\title{
CLASSIFICATION OF DISCRETE SERIES BY MINIMAL $K$-TYPE
}

\author{
RAJAGOPALAN PARTHASARATHY
}

\begin{abstract}
Following the proof by Hecht and Schmid of Blattner's conjecture for $K$ multiplicities of representations belonging to the discrete series it turned out that some results which were earlier known with some hypothesis on the Harish-Chandra parameter of the discrete series representation could be extended removing those hypotheses. For example this was so for the geometric realization problem. Occasionally a few other results followed by first proving them for Harish-Chandra parameters which are sufficiently regular and then using Zuckerman translation functors, wall crossing methods, etc. Recently, Hongyu He raised the question (private communication) of whether the characterization of a discrete series representation by its lowest $K$-type, which was proved by this author and R. Hotta with some hypothesis on the HarishChandra parameter of the discrete series representations, can be extended to all discrete series representations excluding none, using a combination of these powerful techniques. In this article we will answer this question using Dirac operator methods and a result of Susana Salamanca-Riba.
\end{abstract}

\section{Preliminaries And Statement of the main theorem}

Let $G^{e}$ be a connected linear real semisimple Lie group whose complexification is simply connected. We assume that $G^{e}$ admits discrete series (which implies that $G^{e}$ has a compact Cartan subgroup). In Sections 1, 2 and 3 of this paper we write $G$ instead of $G^{e}$; in Section $4 G$ will not be assumed to be connected and $G^{e}$ will denote the identity component. Let $T$ be a compact Cartan subgroup of $G$. Let $K$ be a maximal compact subgroup of $G$ containing $T$. Denote by $\mathfrak{t}, \mathfrak{g}, \mathfrak{k}$ the corresponding Lie subalgebras and $\mathfrak{t}^{\mathbb{C}}, \mathfrak{g}^{\mathbb{C}}, \mathfrak{k}^{\mathbb{C}}$ their complexifications. Let $\mathfrak{g}^{\mathbb{C}}=\mathfrak{k}^{\mathbb{C}}+\mathfrak{p}^{\mathbb{C}}$ be the Cartan decomposition. Here $\mathfrak{p}$ is the orthogonal complement of $\mathfrak{k}$ in $\mathfrak{g}$. Fix a positive system $P$ in the set of roots $\Delta\left(\mathfrak{g}^{\mathbb{C}}, \mathfrak{t}^{\mathbb{C}}\right)$. The set of roots $\Delta\left(\mathfrak{k}^{\mathbb{C}}, \mathfrak{t}^{\mathbb{C}}\right)$ is a subset of $\Delta\left(\mathfrak{g}^{\mathbb{C}}, \mathfrak{t}^{\mathbb{C}}\right)$. Intersecting $P$ with $\Delta\left(\mathfrak{k}^{\mathbb{C}}, \mathfrak{t}^{\mathbb{C}}\right)$ we get a positive system $P_{\mathfrak{k}}$ of $\Delta\left(\mathfrak{k}^{\mathbb{C}}, \mathfrak{t}^{\mathbb{C}}\right)$. The complement of $P_{\mathfrak{k}}$ in $P$ is denoted $P_{n}$ and is called the set of noncompact positive roots. The root spaces $\mathfrak{g}^{\alpha}$ for $\alpha \in P_{n} \bigcup-P_{n}$ span $\mathfrak{p}^{\mathbb{C}}$. Let $\lambda \in \mathfrak{t}_{\mathbb{R}}^{*}=$ Hom $(\sqrt{-1} \mathfrak{t}, \mathbb{R})$ be a $P$-dominant integral linear form. Denote by $\rho$ half the sum of the roots in $P$, by $\rho_{\mathfrak{k}}$ half the sum of the roots in $P_{\mathfrak{k}}$ and by $\rho_{n}$ half the sum of the roots in $P_{n}$. Then $\lambda+\rho$ is $P$-dominant regular integral and $\rho_{n}$ is $P_{\mathfrak{k}}$-dominant

Received by the editors July 29, 2014 and, in revised form, December 4, 2014 and August 14, 2015 .

2010 Mathematics Subject Classification. Primary 22E46; Secondary 22D30.

Key words and phrases. Dirac operator inequality, Dirac cohomology, Harish-Chandra class group, Mackey's criterion, $A_{\mathfrak{q}, \lambda}$.

This research was supported by Raja Ramanna Fellowship from DAE.

The author thanks the referee for suggestions to improve the article by addressing the case of general groups of Harish-Chandra class. His comments on the initial proof of Theorem 1.1 in Section 3 have greatly helped in adding considerable clarity to the original submission. 
integral. Let $\Lambda=\lambda+\rho$. Denote by $\varpi(\Lambda)$ an irreducible unitary representation of $G$ belonging to the discrete series representation with Harish-Chandra parameter $\Lambda$. The restriction $\varpi(\Lambda) \mid K$ of $\varpi(\Lambda)$ to $K$ decomposes as an orthogonal sum of finitedimensional irreducible modules for $K$. In this restriction the finite-dimensional $K$-representation $\tau_{\lambda+2 \rho_{n}}$ with $P_{\mathfrak{k}}$-highest weight $\lambda+2 \rho_{n}$ occurs and $\tau_{\mu}$ does not occur if $\mu$ is $P_{\mathfrak{k}}$-dominant and $\mu=\lambda+2 \rho_{n}-\alpha$ where $\alpha \in P_{n}$.

Our main result is the following.

1.1. Theorem. Let $\pi$ be an irreducible unitary representation of $G$. Suppose that

(i) the finite-dimensional $K$-representation $\tau_{\lambda+2 \rho_{n}}$ with $P_{\mathfrak{k}}$ - highest weight $\lambda+$ $2 \rho_{n}$ occurs in $\left.\pi\right|_{K}$; and

(ii) $\tau_{\mu}$ does not occur in $\left.\pi\right|_{K}$ if $\mu$ is $P_{\mathfrak{k}}$-dominant and $\mu=\lambda+2 \rho_{n}-\alpha$ where $\alpha \in P_{n}$.

Then $\pi$ is unitarily equivalent to the discrete series representation $\varpi(\Lambda)$.

It may be worthwhile to rephrase the above conclusion in a slightly different way (leaving no room to doubt whether a certain assumption is vacuously satisfied).

1.1.1. 'Companion' to Theorem 1.1. Let $\pi$ be an irreducible unitary representation of $G$. Suppose that the finite-dimensional $K$-representation $\tau_{\lambda+2 \rho_{n}}$ with $P_{\mathfrak{k}}$ highest weight $\lambda+2 \rho_{n}$ occurs in $\left.\pi\right|_{K}$; then one of the following cases occurs:

Case (i) There is no $\alpha \in P_{n}$ such that $\lambda+2 \rho_{n}-\alpha$ is $P_{\mathfrak{k}}$-dominant. In this case $\pi \simeq \varpi(\Lambda)$.

Case (ii) There is an $\alpha \in P_{n}$ such that $\lambda+2 \rho_{n}-\alpha$ is $P_{\mathfrak{k}}$-dominant; but no $P_{\mathfrak{k}-}$ highest weight of an irreducible constituent of of $\left.\pi\right|_{\mathfrak{k}}$ is of this form. In this case $\pi \simeq \varpi(\Lambda)$.

Case(iii) There is an $\alpha \in P_{n}$ such that $\lambda+2 \rho_{n}-\alpha$ is $P_{\mathfrak{k}}$-dominant and is the highest weight of an irreducible $\mathfrak{k}$-constituent of $\left.\pi\right|_{\mathfrak{k}}$. In this case $\pi \nsucceq \varpi(\Lambda)$.

After reviewing some preliminary material in the next section we will begin the proof. We remark at the outset that our proof arrives at the conclusion of Theorem 1.1 (and its companion) under a hypothesis slightly weaker than 1.1(ii). More precisely, still under hypothesis 1.1(i)

1.1.2. (i) $\pi \simeq_{G} \varpi(\Lambda)$ if $\tau_{\mu}$ as in Theorem 1.1(ii) does not occur in the $\mathfrak{k}$-span of $\pi(\mathfrak{g})\left(\mathrm{W}_{\lambda+2 \rho_{n}}\right)$ where $\mathrm{W}_{\lambda+2 \rho_{n}}$ is an irreducible $\mathfrak{k}$ submodule of $\left.\pi\right|_{K}$ given by 1.1(i).

1.1.2. (ii) If $\pi \not \varpi \varpi(\Lambda)$, then there is an $\alpha \in P_{n}$ such that $\lambda+2 \rho_{n}-\alpha$ is $P_{\mathfrak{k}}$-dominant and is the highest weight of an irreducible $\mathfrak{k}$-constituent of $\pi(\mathfrak{g})\left(\mathrm{W}_{\lambda+2 \rho_{n}}\right)$.

In Section 4 we discuss to what extent Theorem 1.1 above can be generalized to more general reductive groups $G$ of Harish-Chandra class. This is done from the point of view of inducing representations from an open subgroup of $G$ and using Mackey's irreducibility criterion. Theorem 1.1 will be used for $G^{e}$, the identity component of $G$. So, $G^{e}$ is assumed to be linear. Proposition 4.7 and 4.9 describe a convenient way to classify all the discrete series for $G$ and the canonical $K$-type in a discrete series representation of $G$. The analogue of Theorem 1.1 is Theorem 4.12. In Section 5 we extend Theorem 1.1 to $A_{\mathfrak{q}}(\lambda)$. 


\section{The spin Represention And the Dirac operator}

The restriction of the Killing form of $\mathfrak{g}$ to $\mathfrak{p}$ is positive definite. Note that $\mathfrak{p}$ has even dimension because of the equal rank hypothesis. Let $\mathfrak{s o}(\mathfrak{p})$ be the orthogonal Lie algebra of $\mathfrak{p}$. Let $\operatorname{Cliff}(\mathfrak{p})$ be the Clifford algebra of $\mathfrak{p}$. As $\mathfrak{p}$ has even dimension the complexified Clifford algebra $\operatorname{Cliff}\left(\mathfrak{p}^{\mathbb{C}}\right)$ is isomorphic to a matrix algebra over $\mathbb{C}$. In other words, we have an isomorphism $\operatorname{Cliff}\left(\mathfrak{p}^{\mathbb{C}}\right) \longrightarrow \operatorname{End}(\mathrm{L})$ into the algebra of endomorphisms of a unique simple module $\mathrm{L}$, the spin representation of the complex Clifford algebra. Both $\mathfrak{p}$ and $\mathfrak{s o}(\mathfrak{p})$ are embedded in Cliff( $\mathfrak{p})$ as a vector subspace and as a Lie subalgebra respectively. The action of the Clifford algebra on the spinors $\mathrm{L}$ gives rise to the Clifford multiplication $\epsilon: \mathfrak{p} \otimes \mathrm{L} \longrightarrow \mathrm{L}, \epsilon(X \otimes s)=\epsilon(X) s$ and the spin representation $\sigma: \mathfrak{s o}(\mathfrak{p}) \longrightarrow \operatorname{End}(\mathrm{L})$ and the two half spin representations $\sigma^{ \pm}: \mathfrak{s o}(\mathfrak{p}) \longrightarrow \operatorname{End}\left(\mathrm{L}^{ \pm}\right)$. It should be observed that $\epsilon\left(\mathfrak{p} \otimes \mathrm{L}^{ \pm}\right) \subseteq \mathrm{L}^{\mp}$.

The weights of the adjoint representation $a d: \mathfrak{k} \longrightarrow \mathfrak{s o}(\mathfrak{p})$ are $\left\{\alpha \mid \alpha \in P_{n} \bigcup-P_{n}\right\}$. Denote $\sigma \circ a d, \sigma^{ \pm} \circ$ ad by $\chi, \chi^{ \pm}$, respectively.

For a subset $A \subseteq P,\langle A\rangle$ denotes $\sum_{\alpha \in A} \alpha$. If $\gamma \in \mathfrak{t}_{\mathbb{R}}^{*}, \gamma$ is a weight of $\chi$ if and only if there exists $A \subseteq P_{n}$ such that $\gamma=\rho_{n}-\langle A\rangle$. Then the multiplicity $m_{\chi}(\gamma)$ of $\gamma$ in $\chi$ equals $\#\left\{A \mid A \subseteq P_{n}, \gamma=\rho_{n}-\langle A\rangle\right\}$. We fix notation so that $\gamma$ is a weight of $\chi^{+}\left(\right.$resp. $\left.\chi^{-}\right)$if and only if there exists $A \subseteq P_{n}$ of even(resp. odd) cardinality such that $\gamma=\rho_{n}-\langle A\rangle$. Evidently, $\tau_{\rho_{n}} \subseteq \chi^{+}$. We use the notation $\Delta_{\chi}, \Delta_{\chi^{ \pm}}$for the set of weights of $\chi, \chi^{ \pm}$, respectively. $\Delta_{\chi}$ is the disjoint union of $\Delta_{\chi^{+}}$and $\Delta_{\chi^{-}}$.

Theorem 1.1 was proved by R. Hotta and the author [1] under the hypothesis that:

(i) $(\lambda, \alpha) \neq 0$ for $\alpha \in P_{n}$.

(ii) $(\lambda, \alpha) \geq\left(\rho_{n}-\langle A\rangle, \alpha\right)$ for $\forall A \subseteq P_{n}$ and $\forall \alpha \in P_{\mathfrak{k}}$.

Here $($,$) is the usual scalar product in \mathfrak{t}_{\mathbb{R}}^{*}$ using the Killing form of $\mathfrak{g}$. W. Schmid (21], Lemma 9) had also proved likewise under the hypothesis that $\lambda+\rho$ is sufficiently nonsingular, i.e., for a suitably chosen positive constant $c,(\lambda+\rho, \alpha)>c$, for all positive roots $\alpha \in P$. Schmid also observed ([22], Theorem 3) that if the $\mathfrak{k}$-type of highest weight $\lambda+2 \rho_{n}$ occurs in $\pi$ and no $\mathfrak{k}$-type with highest weight $\lambda+2 \rho_{n}-\langle A\rangle, A \subseteq P_{n}, A \neq \emptyset$ occurs in $\pi$, then $\pi \simeq \varpi(\Lambda)$.

We give a short proof of Theorem 1.1 which uses the formal Dirac operator for an irreducible representation, a result of J.-S. Huang and P. Pandžić about unitary modules with Dirac cohomology ([2], Theorem 6.1) and a result of Susana Salamanca-Riba [1] on the classification of irreducible unitary representations with the same infinitesimal character as an irreducible finite-dimensional representation.

For an irreducible unitary representation $\pi$ of $G$ on a Hilbert space $\mathrm{H}$ let $\mathrm{H}_{\pi}$ be the space of $K$-finite vectors in $\mathrm{H}$. We use the same symbol $\pi$ when referring to $\mathrm{H}_{\pi}$ considered as a $(\mathfrak{g}, K)$ module. The Dirac operator $D_{\pi}: \mathrm{H}_{\pi} \otimes \mathrm{L} \longrightarrow \mathrm{H}_{\pi} \otimes \mathrm{L}$ is defined as $\sum_{i} \pi\left(X_{i}\right) \otimes \epsilon\left(X_{i}\right)$ where $\left\{X_{1}, X_{2}, \cdots, X_{n}\right\}$ is an orthonormal basis of $\mathfrak{p}$. It is independent of the choice of the orthonormal basis.

Let $\mathrm{W}$ be a finite-dimensional subspace of $\mathrm{H}_{\pi}$ stable under $K$. Let $\mathfrak{p}^{\mathbb{C}} \cdot \mathrm{W}$ be the subspace of $\mathrm{H}_{\pi}$ spanned by $\{\pi(X) v \mid X \in \mathfrak{p}, v \in \mathrm{W}\}$.

Remark 2.1.1. We have an obvious $K$-module surjection $\mathfrak{p}^{\mathbb{C}} \otimes \mathrm{W} \longrightarrow \mathfrak{p}^{\mathbb{C}} \cdot \mathrm{W}$.

Remark 2.1.2. Clearly, $D_{\pi}(\mathrm{W} \otimes \mathrm{L}) \subseteq\left(\mathfrak{p}^{\mathbb{C}} \cdot \mathrm{W}\right) \otimes \mathrm{L}$.

2.2. Lemma. Let $\pi$ be an irreducible unitary representation of $G$. Let $\mathrm{W}_{\lambda+2 \rho_{\mathrm{n}}}$ be a $K$-stable subspace of $\mathrm{H}_{\pi}$ such that the $K$-module $\mathrm{W}_{\lambda+2 \rho_{\mathrm{n}}}$ is irreducible and is 
isomorphic to the finite-dimensional $K$ representation $\tau_{\lambda+2 \rho_{n}}$ with $P_{\mathfrak{k}}$-highest weight $\lambda+2 \rho_{n}$. Then:

(i) $\exists$ a k-stable subspace $\mathrm{W}_{\lambda+\rho_{\mathrm{n}}}$ of $\mathrm{W}_{\lambda+2 \rho_{\mathrm{n}}} \otimes \mathrm{L}$ which is an irreducible $\mathfrak{k}$-module with $P_{\mathfrak{k}}$-highest weight $\lambda+\rho_{n}$.

(ii) In Case (i) and Case (ii) of 1.1 .1 if $\mu$ is the $P_{\mathfrak{k}}$-highest weight of an irreducible $\mathfrak{k}$-submodule of $D_{\pi}\left(\mathrm{W}_{\lambda+2 \rho_{\mathrm{n}}} \otimes \mathrm{L}\right)$, then $\mu \neq \lambda+\rho_{n}$.

Remark 2.2.1. For the proof of the lemma above and corollary below we need only the weaker hypothesis ' $\tau_{\mu}$ of (ii) in Theorem 1.1 does not occur in $\mathfrak{p}^{\mathbb{C}} \cdot \mathrm{W}_{\lambda+2 \rho_{n}}$ '.

2.3. Corollary. Let $\mathrm{W}_{\lambda+\rho_{\mathrm{n}}}$ be as in Lemma 2.2. Then in Case (i) and Case (ii) of 1.1.1 $D_{\pi}\left(\mathrm{W}_{\lambda+\rho_{\mathrm{n}}}\right)=0$.

The corollary is an immediate consequence of 2.2 (ii).

Proof of Lemma 2.2. We have $\tau_{\lambda+2 \rho_{n}} \subseteq_{\mathfrak{k} \mathbb{C}} \tau_{\lambda+\rho_{n}} \otimes \tau_{\rho_{n}}$. Also, $\tau_{\rho_{n}} \subseteq_{\mathfrak{k} \mathbb{C}} \chi$ and $\chi$ is self-dual. Thus $\tau_{\lambda+\rho_{n}} \subseteq_{\mathfrak{k C}} \tau_{\lambda+2 \rho_{n}} \otimes \chi$. This proves part (i) of the lemma.

Let $\mathrm{W}_{\lambda+2 \rho_{n}}$ be as in Lemma 2.2. Any irreducible component of $\mathfrak{p}^{\mathbb{C}} \otimes \mathrm{W}_{\lambda+2 \rho_{n}}$ is of the form $\tau_{\mu}$ for some $\mu=\lambda+2 \rho_{n} \pm \alpha$ where $\alpha \in P_{n}$. In Case (i) and Case (ii) of 1.1.1 for $\alpha \in P_{n}, \lambda+2 \rho_{n}-\alpha$ cannot be the highest weight $\mu$ of an irreducible component of $\mathfrak{p}^{\mathbb{C}} \cdot \mathrm{W}_{\lambda+2 \rho_{n}}$. Hence, if $\tau_{\mu} \subseteq \mathfrak{p}^{\mathbb{C}} \cdot \mathrm{W}_{\lambda+2 \rho_{n}}$, then $\mu=\lambda+2 \rho_{n}+\alpha$ for some $\alpha \in P_{n}$. Hence, if $\xi$ is the $P_{\mathfrak{k}}$-highest weight of an irreducible component $\tau_{\xi}$ of $\left(\mathfrak{p}^{\mathbb{C}} \cdot \mathrm{W}_{\lambda+2 \rho_{n}}\right) \otimes \mathrm{L}$, then $\xi=\lambda+2 \rho_{n}+\alpha+\rho_{n}-\langle A\rangle$ for some $\alpha \in P_{n}$ and $A \subseteq P_{n}$. So if $A^{c}$ is the complement of $A$ in $P_{n}$, then $\xi=\lambda+2 \rho_{n}+\alpha+\rho_{n}-\langle A\rangle=\lambda+2 \rho_{n}+\alpha+\rho_{n}-\left\langle P_{n}\right\rangle+\left\langle A^{c}\right\rangle=$ $\lambda+2 \rho_{n}+\alpha+\rho_{n}-2 \rho_{n}+\left\langle A^{c}\right\rangle=\lambda+\alpha+\rho_{n}+\left\langle A^{c}\right\rangle$. Hence, $\xi+\rho_{\mathfrak{k}}=\lambda+\rho+\alpha+\left\langle A^{c}\right\rangle$. In particular, since $\lambda+\rho$ is nonsingular and $P$-dominant then $\left(\xi+\rho_{\mathfrak{k}}, \xi+\rho_{\mathfrak{k}}\right)$ is strictly greater than $\left(\lambda+\rho_{n}+\rho_{\mathfrak{k}}, \lambda+\rho_{n}+\rho_{\mathfrak{k}}\right)$. Consequently, such a $\xi$ cannot equal $\lambda+\rho_{n}$. Since $D_{\pi}\left(\mathrm{W}_{\lambda+2 \rho_{n}} \otimes \mathrm{L}\right) \subseteq\left(\mathfrak{p}^{\mathbb{C}} \cdot \mathrm{W}_{\lambda+2 \rho_{n}} \otimes \mathrm{L}\right)$ it follows that if $\xi$ is the $P_{\mathfrak{k}}$-highest weight of an irreducible component $\tau_{\xi}$ of $D_{\pi}\left(\mathrm{W}_{\lambda+2 \rho_{n}} \otimes \mathrm{L}\right)$, then such a $\xi$ cannot equal $\lambda+\rho_{n}$.

This proves part(ii) of Lemma 2.2 .

\section{The PRoof of Theorem 1.1}

To apply Lemma 2.2 and Corollary 2.3 for the proof of Theorem 1.1 we need to understand irreducible unitary representations with Dirac cohomology. This is the subject of $\S 6$ in the paper [2] of J.-S. Huang and Pandžić. We use a theorem of J.-S. Huang and Pandžić ([2], Theorem 6.1). The short proof of their Theorem 6.1, 2] relies on their Theorem 2.3, 2] proving a conjecture of D. Vogan: if an irreducible finite-dimensional $\mathfrak{k}$-module $\tau_{\xi}$ with $P_{\mathfrak{k}}$-highest weight $\xi$ occurs in the kernel of the Dirac operator $D_{\pi}$ for an irreducible unitary representation $\pi$ of $G$ the infinitesimal character of $\pi$ is given $\xi+\rho_{\mathfrak{k}}$. The referee has pointed out that inasmuchas we appeal only to Theorem 6.1, 2 2 the current paper does not use Vogan's conjecture in an essential way. The referee has further pointed out that for our purposes the techniques of Vogan-Zuckerman ([18, [2], Theorem 5.3) and Kumaresan [20] suffice. Huang and Pandžić also point out below in the statement of their Theorem 6.1, 2] that for its proof the same approach as Vogan-Zuckerman's generalization [18] of Kumaresan's result [20] would suffice, but not without some more labor (one or two more pages). Note that to proceed further we have to exhibit the infinitesimal character of the irreducible unitary representation $\pi$ of Theorem 1.1. In the Dirac cohomology approach of Huang and Pandžić which suggests itself, 
in view of Corollary 2.3, the infinitesimal character can be readily recognized from Vogan's conjecture which has now been a theorem for long.

Since $\xi=\lambda+\rho_{n}$ is in the Dirac kernel of our $\pi$ (from Corollary 2.3) the infinitesimal character of $\pi$ is given by the orbit of $\lambda+\rho$. Let $\alpha_{1}, \alpha_{2}, \cdots, \alpha_{i}$ be all the positive noncompact roots on which $\lambda$ vanishes.

3.1. Then using Salamanca-Riba [11, Theorem 6.1 of [2] concludes that there exists a $\theta$-stable parabolic subalgebra $\mathfrak{q}=\mathfrak{l}+\mathfrak{u}$ containing the Borel subalgebra $\mathfrak{b}$ corresponding to the positive system $P$ such that

$$
P_{n} \cap \Delta\left(\mathfrak{l}^{\mathbb{C}}, \mathfrak{t}^{\mathbb{C}}\right) \subseteq\left\{\alpha_{1}, \alpha_{2}, \cdots, \alpha_{i}\right\} \quad \text { and } \quad \pi \simeq A_{\mathfrak{q}}(\lambda) .
$$

These modules are variously referred to as 'generalized Enright-Varadarajan modules', 'derived functor modules', 'cohomologically induced modules' and 'modules with nonzero relative Lie algebra cohomology' ([10, [18, [19]). The main properties of these modules can be found in these publications and also [2, Theorem 5.3. Among the most notable properties the ones relevant to us here are

3.1 (i) The restriction of $A_{\mathfrak{q}}(\lambda)$ to $\mathfrak{k}$ contains the representation of $\mathfrak{k}$ of the highest weight $\lambda+2 \rho\left(\mathfrak{u} \cap \mathfrak{p}^{\mathbb{C}}\right)$. This weight is the same as $\lambda+\left\langle\Delta\left(\mathfrak{u} \cap \mathfrak{p}^{\mathbb{C}}\right)\right\rangle$ in earlier notation (third paragraph, §2).

3.1 (ii) If the representation of $\mathfrak{k}$ of the highest weight $\delta$ occurs in $A_{\mathfrak{q}}(\lambda)$, then

$$
\delta=\lambda+2 \rho\left(\mathfrak{u} \cap \mathfrak{p}^{\mathbb{C}}\right)+\sum_{\beta \in \Delta\left(\mathfrak{u} \cap \mathfrak{p}^{\mathbb{C}}\right)} n_{\beta} \beta
$$

with $n_{\beta}$ nonnegative integers.

Since the representation of $\mathfrak{k}$ with highest weight $\lambda+2 \rho_{n}$ occurs in $\pi \simeq A_{\mathfrak{q}}(\lambda)$ we note that $\lambda+2 \rho_{n}=\lambda+2 \rho\left(\mathfrak{u} \cap \mathfrak{p}^{\mathbb{C}}\right)+\sum_{\beta \in \Delta\left(\mathfrak{u} \cap \mathfrak{p}^{\mathbb{C}}\right)} n_{\beta} \beta$ with $n_{\beta}$ nonnegative integers. So,

$$
\begin{aligned}
2 \rho_{n}-2 \rho\left(\mathfrak{u} \cap \mathfrak{p}^{\mathbb{C}}\right) & =\sum_{\beta \in \Delta\left(\mathfrak{u} \cap \mathfrak{p}^{\mathbb{C}}\right)} n_{\beta} \beta, \quad \text { i.e., } \\
\left\langle P_{n} \cap \Delta(\mathfrak{l})\right\rangle & =\sum_{\beta \in \Delta\left(\mathfrak{u} \cap \mathfrak{p}^{\mathbb{C}}\right)} n_{\beta} \beta .
\end{aligned}
$$

Evaluating both sides of the last equation on the center of $\mathfrak{l}$, while the left side evaluates to zero, the right side would not evaluate to zero unless $n_{\beta}=0, \forall \beta \in$ $\Delta\left(\mathfrak{u} \cap \mathfrak{p}^{\mathbb{C}}\right)$. Hence

$$
\lambda+2 \rho_{n}=\lambda+2 \rho\left(\mathfrak{u} \cap \mathfrak{p}^{\mathbb{C}}\right) \Longrightarrow 2 \rho_{n}=2 \rho\left(\mathfrak{u} \cap \mathfrak{p}^{\mathbb{C}}\right) \Longrightarrow \Delta\left(\mathfrak{u} \cap \mathfrak{p}^{\mathbb{C}}\right)=P_{n} .
$$

Thus, we conclude that $\pi=A_{\mathfrak{q}}(\lambda)=A_{\mathfrak{b}}(\lambda) \simeq \varpi(\Lambda)$.

This completes the proof of Theorem 1.1.

For convenience of later reference (in 4.11 and 4.13.4) we prove a property of discrete series representations, for which we do not know a reference. (In this section $G$ is connected. In the next section $G$ is disconnected; we will apply the following fact in the next section to the idenity component $G^{e}$ of $G$.) Let $\varpi(\Lambda)$ be a discrete series representation for $G$ with indicated Harish-Chandra parameter. We use other notation already introduced. So $\Lambda$ is $P$ dominant regular integral and $\Lambda=\lambda+\rho$. As already noted the irreducible $K$ module $\tau_{\lambda+2 \rho_{n}}$ with $P_{\mathfrak{k}}$ highest weight $\lambda+2 \rho_{n}$ occurs in $\left.\varpi(\Lambda)\right|_{K}$ with multiplicity 1 . 
Proposition 3.2. If $\alpha \in P_{n}$ and $\lambda+2 \rho_{n}-\alpha$ is $P_{\mathfrak{k}}$ dominant, then $\tau_{\lambda+2 \rho_{n}-\alpha}$ does not occur in $\left.\varpi\left(\Lambda^{\prime}\right)\right|_{K}$ for any discrete series representation $\varpi\left(\Lambda^{\prime}\right)$ for which $\left|\Lambda^{\prime}\right| \geq|\Lambda|$.

The proof we outline here in fact applies without change to show the following more general assertion.

Proposition 3.2.0. For a nonempty subset $A \subseteq P_{n}$ let $\langle A\rangle=\sum_{\alpha \in A} \alpha$. Suppose that $\lambda+2 \rho_{n}-\langle A\rangle$ is $P_{\mathfrak{k}}$ dominant. Then $\tau_{\lambda+2 \rho_{n}-\langle A\rangle}$ does not occur in $\left.\varpi\left(\Lambda^{\prime}\right)\right|_{K}$ for any discrete series representation $\varpi\left(\Lambda^{\prime}\right)$ for which $\left|\Lambda^{\prime}\right| \geq|\Lambda|$.

(Remark: $\Lambda^{\prime}$ and $\Lambda$ may be dominant with respect to different positive systems.)

Proof of 3.2. We will derive it as an application of Dirac inequality for the noncompact symmetric space as well as the dual compact symmetric space ([9], Proposition 2.6) and ([8, Lemma 8.1).

The $P_{\mathfrak{k}}$ highest weight of the PRV component ([7, Lemma 2.26, 15]) and ([16], Theorem 3.5 and $\S 3.2$ ) of $\tau_{1} \otimes \tau_{2}$ lies in the $W_{K}$-orbit of the sum 'highest weight of $\tau_{1}+$ lowest weight of $\tau_{2}$ '. According to V.S. Varadarajan from his excellent reminiscences [15] of the historical evolution of [7] (especially [15], pp. xiv and xv) the PRV component should really have been called PRVV component to underline the important role of S.R.S. Varadhan.

For any discrete series representation $\varpi\left(\Lambda_{1}\right)$ of $G$ we have the following fact about when the $P_{\mathfrak{k}}$ highest weight of an irreducible $\mathfrak{k}$ constituent in $\mathrm{H}_{\varpi\left(\Lambda_{1}\right)} \otimes \mathrm{L}$ is minimal.

Fact 3.2.1. Suppose $\left.\tau_{\mu_{1}} \subseteq_{\mathfrak{k}} \varpi\left(\Lambda_{1}\right)\right|_{\mathfrak{k}}, \tau_{\mu_{2}} \subseteq_{\mathfrak{k}} \mathrm{L}$ and $\tau_{\zeta} \subseteq_{\mathfrak{k}} \tau_{\mu_{1}} \otimes \tau_{\mu_{2}}$.

Then $\left|\zeta+\rho_{\mathfrak{k}}\right| \geq\left|\Lambda_{1}\right|$. Equality holds if and only if

(a) there exists a positive system $P^{\circ} \subseteq \Delta\left(\mathfrak{g}^{\mathbb{C}}, \mathfrak{t}^{\mathbb{C}}\right)$ containing $P_{\mathfrak{k}}$ such that $\left(\Lambda_{1}, \alpha\right)>0, \forall \alpha \in P^{\circ}$,

(b) $\Lambda_{1}=\mu_{1}-\rho_{n}^{\circ}+\rho_{\mathfrak{k}}$, where $\rho_{n}^{\circ}=\frac{1}{2} \sum_{\alpha \in P_{n}^{\circ}} \alpha$ and $\rho^{\circ}=\frac{1}{2} \sum_{\alpha \in P^{\circ}} \alpha$,

(c) the lowest weight of $\tau_{\mu_{2}}$ is $-\rho_{n}^{\circ}$, and

(d) $\tau_{\zeta}$ is the PRV component of $\tau_{\mu_{1}} \otimes \tau_{\mu_{2}}$.

When equality holds,

(e) the sum "highest weight of $\tau_{\mu_{1}}+$ the lowest weight of $\tau_{\mu_{2}}$ " is already $P_{\mathfrak{k}}$ dominant and equals $\zeta$.

(f) (c) above implies that $\mu_{2}$ determines $P^{\circ}$ in (a): If $\gamma$ is the $P_{\mathfrak{k}}$ highest weight of $\tau_{\mu_{2}}^{*}$, then $P^{\circ}=\left\{\alpha \in \Delta\left(\mathfrak{g}^{\mathbb{C}}, \mathfrak{t}^{\mathbb{C}}\right) \mid\left(\gamma+\rho_{\mathfrak{k}}, \alpha\right)>0\right\}$.

We apply this to prove Proposition 3.2. Suppose $\tau_{\lambda+2 \rho_{n}-\alpha}$ occurs in $\left.\varpi\left(\Lambda^{\prime}\right)\right|_{K}$. Consider the PRV component in the tensor product $\tau_{\lambda+2 \rho_{n}-\alpha} \otimes \tau_{\rho_{n}}^{*}$. Denote by $\xi$ the $P_{\mathfrak{k}}$ dominant linear form in the $W_{K^{-}}$orbit of $\lambda+\rho_{n}-\alpha$. Then $\tau_{\xi}$ is the PRV component in $\tau_{\lambda+2 \rho_{n}-\alpha} \otimes \tau_{\rho_{n}}^{*}$. Here $\tau_{\rho_{n}}^{*}$ is the representation contragredient to $\tau_{\rho_{n}}$. $\tau_{\rho_{n}}$ has highest weight $\rho_{n}, \tau_{\rho_{n}} \subseteq_{\mathfrak{k}} \mathrm{L}$ (the total spin module which is self-dual). So $\tau_{\rho_{n}}^{*} \subseteq_{\mathfrak{k}} \mathrm{L}$. Also, $\tau_{\rho_{n}}^{*}$ has lowest weight $-\rho_{n}$. Thus $\tau_{\xi}$ occurs in $\varpi\left(\Lambda^{\prime}\right) \otimes \mathrm{L}$. Hence we have the Dirac inequality $\left|\Lambda^{\prime}\right| \leq\left|\xi+\rho_{\mathfrak{k}}\right|$. But by hypothesis, $|\Lambda| \leq\left|\Lambda^{\prime}\right|$. So $|\Lambda| \leq\left|\xi+\rho_{\mathfrak{k}}\right|$.

Denote the irreducible finite-dimensional $\mathfrak{g}^{\mathbb{C}}$ module with $P$ highest weight $\lambda$ by $\mathrm{F}_{\lambda}$. As $\lambda+\rho_{n}-\alpha$ is a weight of $\mathrm{F}_{\lambda} \otimes \mathrm{L}$ from (8, Lemma 8.1, which can be viewed as Dirac inequality for the compact dual symmetric space) and (4], Lemma 5.8), 
$|\Lambda| \geq\left|\xi+\rho_{\mathfrak{k}}\right|$. Hence

$$
|\Lambda|=\left|\xi+\rho_{\mathfrak{k}}\right|=\left|\Lambda^{\prime}\right| \text {. }
$$

In view of this equality, to draw conclusions using in particular 3.2.1(e) and (f) we use the comparisons below:

$\begin{array}{ccc}3.2 .1 & \leftrightarrow & 3.2 \\ \tau_{\mu_{1}} & \leftrightarrow & \tau_{\lambda+2 \rho_{n}-\alpha} \\ \tau_{\mu_{2}} & \leftrightarrow & \tau_{\rho_{n}}^{*} \\ \text { PRV } & \leftrightarrow & \operatorname{PRV} \xi \\ -\rho_{n}^{\circ} \text { is } & & -\rho_{n} \text { is } \\ \text { lowest weight of } \tau_{\mu_{2}} & \leftrightarrow & \text { lowest weight of } \tau_{\rho_{n}}^{*} \\ \rho_{n}^{\circ} & \leftrightarrow & \rho_{n} \\ \rho^{\circ} & \leftrightarrow & P \\ P^{\circ} & \leftrightarrow & \varpi\left(\Lambda^{\prime}\right) \\ \varpi\left(\Lambda_{1}\right) & \leftrightarrow & \Lambda^{\prime}=\left(\lambda+2 \rho_{n}-\alpha\right)-\rho_{n}+\rho_{\mathfrak{k}} \\ \Lambda_{1}=\mu_{1}-\rho_{n}^{\circ}+\rho_{\mathfrak{k}} & \leftrightarrow & \Lambda^{\prime}=\left(\Lambda^{\prime} \text { is } P \text { dominant }\right. \\ \Lambda_{1} \text { is } P^{\circ} \text { dominant } & \leftrightarrow & \end{array}$

Applying 3.2.1 we conclude that: (i) the $P_{\mathfrak{k}}$ dominant linear form in the $W\left(\mathfrak{k}^{\mathbb{C}}, \mathfrak{t}^{\mathbb{C}}\right)$ orbit of $\lambda+\rho_{n}-\alpha$ is $\lambda+\rho_{n}-\alpha$ itself. (ii) ' $\rho_{n}^{\circ}$ ' of the discussion in 3.2.1 worked out in this context is $\rho_{n}, \rho^{\circ}=\rho_{\mathfrak{k}}+\rho_{n}^{\circ}$ then becomes $\rho_{\mathfrak{k}}+\rho_{n}$, giving rise to $P^{\circ}=P$, (iii) $\left(\Lambda^{\prime}, \alpha\right)>0, \forall \alpha \in P^{\circ}$ which implies $\left(\Lambda^{\prime}, \alpha\right)>0, \forall \alpha \in P$ as $P^{\circ}=P$; but $\Lambda^{\prime}=\lambda+\rho-\alpha=\Lambda-\alpha$ from the comparison table above. So, $(\Lambda, \Lambda)=\left(\Lambda^{\prime}+\alpha, \Lambda^{\prime}+\right.$ $\alpha)=\left(\Lambda^{\prime}, \Lambda^{\prime}\right)+2\left(\Lambda^{\prime}, \alpha\right)+(\alpha, \alpha)$ which implies $|\Lambda|>\left|\Lambda^{\prime}\right|$ which contradicts (3.2.2).

This completes the proof of Lemma 3.2 .

\section{Disconnected $G$}

In this section we discuss briefly to what extent we can generalize Theorem 1.1 to more general real reductive groups $G$ of Harish-Chandra class. Let $G^{e}$ be the identity component of $G$. $G$ satisfies the following axioms.

4.1 (a) $G$ has finitely many connected components.

4.1 (b) There exists a Lie group homomorphism $\Psi: G \longrightarrow \mathrm{I}\left(\mathfrak{g}^{\mathbb{C}}\right)$ such that for $y \in G, \operatorname{Ad}_{G}(y): \mathfrak{g} \rightarrow \mathfrak{g}$ is given by $\left.\operatorname{Ad}_{\mathrm{I}\left(\mathfrak{g}^{\mathbb{C}}\right)} \Psi(y)\right|_{\mathfrak{g}}$. Here $\mathrm{I}\left(\mathfrak{g}^{\mathbb{C}}\right)$ is the connected complex adjoint group of inner automorphisms of the Lie algebra $\mathfrak{g}^{\mathbb{C}}$ which is a subgroup of $G L\left(\mathfrak{g}^{\mathbb{C}}\right)$.

4.1 (c) If $\mathfrak{g}=\mathfrak{c} \oplus \mathfrak{g}_{1}$ is the decomposition of $\mathfrak{g}$ into center and semisimple part the analytic subgroup $G_{1}$ of $G^{e}$ with Lie algebra $\mathfrak{g}_{1}$ has finite center.

These axioms were introduced by Harish-Chandra in [5] and [6].

We will restrict to the case where $G_{1}$ is linear and has a compact Cartan subgroup and the center of the identity component $G^{e}$ of $G$ has trivial split component. This implies that $G^{e}$ has compact center.

Every $X \in \mathfrak{g}^{\mathbb{C}}$ gives rise to a left $G$-invariant vector field $l_{X}$ on $G$ and also a right $G$-invariant vector field $\mathbf{r}_{X}$. Every $u \in U\left(\mathfrak{g}^{\mathbb{C}}\right.$ ) (enveloping algebra) gives rise to a left(right) invariant differential operator on $G$.

4.2. Since $G$ need not be connected an element $z$ of the center of $U(\mathfrak{g})$ may not in general give rise to an 'Ad' invariant differential operator on $G$. But the hypothesis 4.1 above implies that $z$ indeed gives rise to an 'Ad' invariant differential operator on $G$. 
Let $\mathrm{I}\left(\mathfrak{g}_{1}^{\mathbb{C}}\right)$ (resp. $\mathrm{I}\left(\mathfrak{g}_{1}\right)$ ) be the group of inner automorphisms of $\mathfrak{g}_{1}^{\mathbb{C}}$ (resp. $\mathfrak{g}_{1}$ ). The following interesting fact was pointed out by B. Kostant during his lectures in TIFR, Bombay in 1969. However, we will not use this in our arguments below, though we were inspired by Kostant's observation.

4.2.1. Remark. Let $\mathrm{I}\left(\mathfrak{g}_{1}\right)_{\theta}=\left\{h \in \mathrm{I}\left(\mathfrak{g}_{1}^{\mathbb{C}}\right) \mid \operatorname{Ad}_{\mathrm{I}\left(\mathfrak{g}_{1}^{\mathbb{C}}\right)}(h) \mathfrak{g}_{1}=\mathfrak{g}_{1}\right\}$. Let $\mathfrak{a}_{1}$ be a maximal $\mathbb{R}$-split abelian subalgebra of $\mathfrak{g}_{1}\left(\forall X \in \mathfrak{a}_{1}, a d_{\mathfrak{g}_{1}}(X): \mathfrak{g}_{1} \rightarrow \mathfrak{g}_{1}\right.$ is diagonalizable having all eigenvalues real). Let $F$ be the finite subgroup of elements of order utmost 2 in the compact torus in $I\left(\mathfrak{g}_{1}^{\mathbb{C}}\right)$ having Lie subalgebra $\sqrt{-1} \mathfrak{a}_{1}$. Then $I\left(\mathfrak{g}_{1}\right)_{\theta}=$ $\mathrm{I}\left(\mathfrak{g}_{1}\right) F$.

4.3. Let $C$ be the center of $G$. Since $G$ need not be connected in general the center $C^{\circ}$ of $G^{e}$ may be bigger than $C \cap G^{e}$. However, in view of 4.1, $C^{\circ}=C \cap G^{e}$.

Let $B$ equal the centralizer of $G^{e}$ in $G$. For a general group $G$ of Harish-Chandra class an element $g \in G$ may centralize $G^{e}$ without being in the center of $G$. Note that $\Psi(B)=\Psi\left(e_{G}\right)$. So $B=\Psi^{-1}\left(\Psi\left(e_{G}\right)\right)$.

4.4. Both $B G^{e}$ and $C G^{e}$ are normal subgroups of $G$ and each is a union of connected components ( $G^{e}$-cosets) of $G$. We have $G \supseteq B G^{e} \supseteq C G^{e} \supseteq G^{e}$. We note $B \cap G^{e}=$ $C^{\circ}=C \cap G^{e}$. Fix a maximal compact subgroup $K_{1} \subseteq G_{1}$ with Lie subalgebra $\mathfrak{k}_{1} \subseteq \mathfrak{g}_{1}$. Define $K=\left\{g \in G \mid g K_{1} g^{-1}=K_{1}\right\}$. Put $K^{e}=K \cap G^{e}$. It is easy to show that $K$ (resp. $K^{e}$ ) is the unique maximal compact subgroup of $G$ (resp. $G^{e}$ ) containing $K_{1}$. We note that $K^{e}$ is the identity component of $K$. Let $\mathfrak{k}$ be the Lie algebra of $K^{e}$. We recall a basic fact about $\mathrm{I}\left(\mathfrak{g}^{\mathbb{C}}\right)$. If an automorphism fixes pointwise each element of a complex Cartan subalgebra of $\mathfrak{g}^{\mathbb{C}}$, then it is given by the conjugation action of an element of the corresponding complex Cartan subgroup. $K^{e}$ has a compact Cartan subgroup of $G^{e}$. So we deduce that if $y \in K$ and $y$ centralizes $K^{e}$, then $\Psi(y)$ belongs to $\Psi(G) \cap \mathcal{Z}$ where $\mathcal{Z}$ is the complexification of a compact Cartan subgroup of $\Psi\left(K^{e}\right)$. This intersection is that compact Cartan subgroup itself. This is so for every compact Cartan subgroup of $\Psi\left(K^{e}\right)$. The intersection of all compact Cartan subgroups of $\Psi\left(K^{e}\right)$ is $\Psi\left(C_{K^{e}}\right)$, where $C_{K^{e}}$ is the center of $K^{e}$. So $\Psi(y) \in \Psi\left(C_{K^{e}}\right)$.

4.4.1. Conclusion. The centralizer of $K^{e}$ in $K$ equals $\Psi^{-1}\left(\Psi\left(e_{G}\right)\right) C_{K^{e}}=B C_{K^{e}}$.

Note that $K \supseteq B K^{e} \supseteq K^{e}$.

The intersection of $K$ and any connected component of $G$ is nonempty using the fact that maximal compact subgroups of $G_{1}$ are $\operatorname{Ad}\left(G_{1}\right)$-conjugate.

Denote by $\theta: \mathfrak{g} \rightarrow \mathfrak{g}$ the Cartan involution corresponding to the Cartan decomposition $\mathfrak{g}=\mathfrak{k}+\mathfrak{p}$. Since we assumed that $G_{1}$ and $K_{1}$ have the same rank, $\theta$ is given by inner conjugation $\operatorname{Ad}_{G}(\theta): \mathfrak{g} \rightarrow \mathfrak{g}$ by an element $\theta \in K_{1}$ (determined uniquely up to group multiplication by an element of $C^{\circ}$ ).

4.5. If $\Gamma, \Gamma^{\prime}$ are open subgroups of $G, \Gamma \subseteq \Gamma^{\prime}$ and $\eta: \Gamma \rightarrow G L\left(V_{\eta}\right)$ is a representation of $\Gamma$ we define as usual the induced representation $\mathcal{I}_{\Gamma}^{\Gamma^{\prime}}\left(V_{\eta}\right)$. In order to avoid cumbersome notation we use the same symbol $\mathcal{I}_{\Gamma}^{\Gamma^{\prime}}\left(V_{\eta}\right)$ both for the induced representation as well as the space on which it acts. So we have

$$
\mathcal{I}_{\Gamma}^{\Gamma^{\prime}}\left(V_{\eta}\right)= \begin{cases}V_{\eta} \text {-valued functions } \phi: \Gamma^{\prime} \rightarrow V_{\eta} & \text { satisfying } \\ \phi(g \gamma)=\eta\left(\gamma^{-1}\right) \phi(g), \forall \gamma \in \Gamma, & \Gamma \text {-equivariant property. }\end{cases}
$$


Group action of $g \in \Gamma^{\prime}$ on functions is by left translation $l_{g}$. When there is no likelihood of confusion we suppress $V_{\eta}$ in the notation for induced representation. For $g, h \in \Gamma^{\prime},\left(\mathcal{I}_{\Gamma}^{\Gamma^{\prime}}(g)\{\phi\}\right)(h)=\left(l_{g} \phi\right)(h)=\phi\left(g^{-1} h\right)$. We have an 'evaluation at unit element' map

$$
\text { ev : }\left\{\begin{array}{l}
V_{\eta} \leftarrow \mathcal{I}_{\Gamma}^{\Gamma^{\prime}}\left(V_{\eta}\right), \\
\phi \mapsto \phi(e) .
\end{array}\right.
$$

If $g \in \Gamma, \phi \in \mathcal{I}_{\Gamma}^{\Gamma^{\prime}}\left(V_{\eta}\right)$ we have $\operatorname{ev}\left(l_{g} \phi\right)=\eta(g)(\operatorname{ev}(\phi))$. We remark that if $\mathcal{W}$ is a nonzero $\Gamma^{\prime}$-submodule of $\mathcal{I}_{\Gamma}^{\Gamma^{\prime}}\left(V_{\eta}\right)$ the evaluation map ev cannot be identically zero on $\mathcal{W}$.

If $\eta_{1}: \Gamma \rightarrow G L\left(V_{1}\right), \eta_{2}: \Gamma \rightarrow G L\left(V_{2}\right)$ are two modules and $\kappa: V_{1} \rightarrow V_{2}$ is a $\Gamma$-module map, then it induces a map $\mathcal{I}(\kappa): \mathcal{I}_{\Gamma}^{\Gamma^{\prime}}\left(V_{1}\right) \rightarrow \mathcal{I}_{\Gamma}^{\Gamma^{\prime}}\left(V_{2}\right)$ commuting with the $\Gamma^{\prime}$ action. Moreover, the following diagram is commutative:

$$
\begin{array}{ccc}
V_{1} & \stackrel{\kappa}{\longrightarrow} & V_{2} \\
\mathbf{e v}_{1} \uparrow & & \mathbf{e v}_{2} \uparrow \\
\mathcal{I}_{\Gamma}^{\Gamma^{\prime}}\left(V_{1}\right) & \stackrel{\mathcal{I}(\kappa)}{\longrightarrow} & \mathcal{I}_{\Gamma}^{\Gamma^{\prime}}\left(V_{2}\right)
\end{array}
$$

4.6. All the constructions and assertions of 4.5 hold if $G$ is replaced by $K$ and $\Gamma, \Gamma^{\prime}$ are open subgroups of $K$. If $E$ is a finite-dimensional $\Gamma^{\prime}$-module there is a natural isomorphism

$$
\begin{gathered}
\mu_{E}: E \otimes \mathcal{I}_{\Gamma}^{\Gamma^{\prime}}(V) \rightarrow \mathcal{I}_{\Gamma}^{\Gamma^{\prime}}(E \otimes V), \\
\left\{\mu_{E}(x \otimes \phi)\right\}(k)=\left(k^{-1} \cdot x\right) \otimes \phi(k),\left(k \in \Gamma^{\prime}\right) .
\end{gathered}
$$

4.6.1. If $\mathcal{W}$ is a $\Gamma^{\prime}$-submodule of $\mathcal{I}_{\Gamma}^{\Gamma^{\prime}}(V)$, then $\operatorname{ev}\left(\mu_{E}(E \otimes \mathcal{W})\right)=E \otimes(\operatorname{ev}(\mathcal{W}))$.

If $\kappa^{*}: E \otimes V \rightarrow V$ is a $\Gamma$-module map and if $\mathcal{W} \subseteq \mathcal{I}_{\Gamma}^{\Gamma^{\prime}}(V)$ is a nonzero $\Gamma^{\prime}$ submodule, then from the commutative diagram

$$
\begin{array}{ccc}
E \otimes V & \stackrel{\kappa^{*}}{\longrightarrow} & V \\
\mathbf{e v}_{(E \otimes V)} \uparrow & & \mathbf{e v}_{(V)} \uparrow \\
\mathcal{I}_{\Gamma}^{\Gamma^{\prime}}(E \otimes V) & \stackrel{\mathcal{I}\left(\kappa^{*}\right)}{\longrightarrow} & \mathcal{I}_{\Gamma}^{\Gamma^{\prime}}(V)
\end{array}
$$

we conclude that

$$
\operatorname{ev}_{(V)}\left\{\mathcal{I}\left(\kappa^{*}\right)\left\langle\mu_{E}(E \otimes \mathcal{W})\right\rangle\right\}=\kappa^{*}\left(E \otimes \mathbf{e v}_{(V)}(\mathcal{W})\right)
$$

Every irreducible finite-dimensional (complex) representation $\tau_{K}$ of $K$ occurs as an irreducible $K$-constituent in a representation $\mathcal{I}_{K^{e}}^{K}\left(\tau_{K^{e}}\right)$ of $K$ obtained by inducing from any irreducible $K^{e}$-constituent $\left.\tau_{K^{e}} \subseteq_{K^{e}}\left(\tau_{K}\right)\right|_{K^{e}}$.

4.6.3. Let $\omega_{G}$ be a discrete series representation of $G$. In view of 4.2 there exists a homomorphism $\chi_{\omega_{G}}: \mathcal{Z}(\mathfrak{g}) \rightarrow \mathbb{C}$ such that for all $z \in \mathcal{Z}(\mathfrak{g})$ and for every $K$-finite matrix coefficient $f: G \rightarrow \mathbb{C}$ of $\omega_{G}, z f=\chi_{\omega_{G}}(z) f$ (see also [17], proof of Lemmas 5.2 and 5.3).

From this it can be deduced that $\left.\left(\omega_{G}\right)\right|_{G^{e}}$ decomposes into a finite sum of discrete series representations of $G^{e}$, all of them having the same infinitesimal character. Furthermore, if $\omega_{1}^{e}, \omega_{2}^{e}$ both occur in $\left.\omega_{G}\right|_{G^{e}}$, then for some $g \in G, \omega_{1}^{e} \simeq_{G^{e}}\left(\omega_{2}^{e}\right)^{g}$, where $\left(\omega_{2}^{e}\right)^{g}(h)=\omega_{2}^{e}\left(g h g^{-1}\right)$ for $h \in G^{e}$. Obviously, if $C$ acts on the representation 
space of $\omega_{G}$ via scalar multiplication by $\xi: C \rightarrow \mathbb{C}^{\times}$, then on each summand of $\left.\omega_{G}\right|_{G^{e}}$ the action of $C^{\circ}$ is given by $\left.\xi\right|_{C^{\circ}}$.

Every irreducible unitary representation $\pi_{G}$ of $G$ occurs as a $G$-summand in a representation $\mathcal{I}_{G^{e}}^{G}\left(\pi_{G^{e}}\right)$ of $G$ obtained by inducing from any irreducible $G^{e}$ summand $\left.\pi_{G^{e}} \subseteq_{G^{e}} \pi_{G}\right|_{G^{e}}$. We note that $\pi_{G}$ belongs to the discrete series of $G$ if and only if $\pi_{G^{e}}$ belongs to the discrete series of $G^{e}$. In that case each irreducible $G$-summand of $\mathcal{I}_{G^{e}}^{G}\left(\pi_{G^{e}}\right)$ belongs to discrete series.

4.6.4. Let $\omega_{G^{e}}$ be a discrete series representation of $G^{e}$. Let $\xi: C \rightarrow \mathbb{C}^{\times}$be a character of $C$ such that $\left.\xi\right|_{C^{\circ}}$ coincides with the action of $\left.\omega_{G^{e}}\right|_{C^{\circ}}$. Let $\nu$ be an irreducible finite-dimensional representation of $B$ such that the action of $\left.\nu\right|_{C}$ is given by $\xi$. Then the tensor product $\nu \otimes \omega_{G^{e}}$ is an irreducible representation of $B \times G^{e}$. It is in fact a discrete series representation of $B \times G^{e}$. Any irreducible unitary representation of $B \times G^{e}$ is of the form $\mu \otimes \pi_{G^{e}}$ where $\mu$ is an irreducible finite dimensional representation of $B$ and $\pi_{G^{e}}$ is an irreducible unitary representation of $G^{e}$. It belongs to the discrete series of $B \times G^{e}$ if and only if $\pi_{G^{e}}$ belongs to the discrete series of $G^{e}$. The subgroup $\left\{\left(z, z^{-1}\right) \mid z \in C^{\circ}\right\}$ of $B \times G^{e}$ is in the kernel of $\nu \otimes \omega_{G^{e}}$. Hence $\nu \otimes \omega_{G^{e}}$ is in fact an irreducible representation of the quotient group $B G^{e}$. Clearly $\nu \otimes \omega_{G e}$ is a discrete series representation of $B G^{e}$ which occurs as a $B G^{e}$-summand in $\mathcal{I}_{G^{e}}^{B G^{e}}\left(\omega_{G^{e}}\right)$, the representation of $B G^{e}$ obtained by inducing from the representation $\omega_{G^{e}}$ of $G^{e}$. Conversely, every irreducible $B G^{e}$-summand $\omega_{B G^{e}}$ of $\mathcal{I}_{G^{e}}^{B G^{e}}\left(\omega_{G^{e}}\right)$ arises in this way as $\nu \otimes \omega_{G^{e}}$ for a suitable choice of an irreducible finite-dimensional representation $\nu$ of $B$. It can be now deduced that every discrete series reresentation of $B G^{e}$ is of the form $\nu \otimes \omega_{G}$ for a suitable choice of a finitedimensional irreducible representation $\nu$ of $B$ and a discrete series representation $\omega_{G^{e}}$ of $G^{e}$.

The induced representation $\mathcal{I}_{G^{e}}^{G}\left(\omega_{G^{e}}\right)$ can be obtained in two stages: first by inducing $\omega_{G^{e}}$ from $G^{e}$ to $B G^{e}$ to get $\mathcal{I}_{G^{e}}^{B G^{e}}\left(\omega_{G^{e}}\right)$ and then inducing the representation $\mathcal{I}_{G^{e}}^{B G^{e}}\left(\omega_{G^{e}}\right)$ from $B G^{e}$ to $G$. After the first stage instead of inducing the entire $\mathcal{I}_{G^{e}}^{B G^{e}}\left(\omega_{G^{e}}\right)$ to $G$ from $B G^{e}$ let us pick a $B G^{e}$-irreducible summand $\nu \otimes \omega_{G^{e}}$ as in 4.6.4 and induce only $\nu \otimes \omega_{G^{e}}$ from $B G^{e}$ to $G$.

4.7. Proposition. The induced representation $\mathcal{I}_{B G^{e}}^{G}\left(\nu \otimes \omega_{G^{e}}\right)$ is irreducible. It is a discrete series representation of $G$. Any discrete series representation of $G$ arises as $\mathcal{I}_{B G^{e}}^{G}\left(\nu \otimes \omega_{G^{e}}\right)$ for a suitable discrete series representation $\omega_{G^{e}}$ of $G^{e}$ and an irreducible finite-dimensional representation $\nu$ of $B$.

Proof. We use a simple version of Mackey's irreducibility criterion for an induced representation. For an exposition of Mackey's criterion there are many sources, for example, 14, $\S 5$. Both $G^{e}$ and $B G^{e}$ are open normal subgroups of $G$ of finite index. According to this irreducibility criterion, $\mathcal{I}_{B G^{e}}^{G}\left(\nu \otimes \omega_{G^{e}}\right)$ is irreducibe if and only if $\forall g \in G \backslash B G^{e}$

$$
\left\{=\text { Latex ' } \$ \mathrm{G} \backslash \text { setminus } B G^{\wedge} \mathrm{e} \$ \text { ' }\right\}
$$

the representations $\nu \otimes \omega_{G^{e}}$ and $\left(\nu \otimes \omega_{G^{e}}\right)^{g}$ are nonisomorphic. Here, $\left(\nu \otimes \omega_{G^{e}}\right)^{g}$ is the representation of $B G^{e}$ on the same Hilbert space as the one on which $\nu \otimes \omega_{G^{e}}$ acts with operators defined by $\left(\nu \otimes \omega_{G^{e}}\right)^{g}(x)=\left(\nu \otimes \omega_{G^{e}}\right)\left(g x g^{-1}\right), \forall x \in B G^{e}$. For $g \in G$, we define a representation $\omega_{G^{e}}^{g}$ of $G^{e}$ in the same way: for $x \in G^{e}, \omega_{G^{e}}^{g}(x)=$ $\omega_{G^{e}}\left(g x g^{-1}\right)$. The group $B$, being the centralizer of $G^{e}$ in $G$, is itself a normal subgroup of $G$ and we define $\nu^{g}$ similarly. Then it is seen that $\left(\nu \otimes \omega_{G^{e}}\right)^{g}=\nu^{g} \bigotimes \omega_{G^{e}}^{g}$. 
We will in fact show that for $g \in G \backslash B G^{e}$ the two representations $\omega_{G^{e}}$ and $\omega_{G^{e}}^{g}$ are nonisomorphic. Observe that the isomorphism class of $\omega_{G^{e}}^{g}$ depends only on the $G^{e}$-coset of $g$. Since $K^{e}$ and $g K^{e} g^{-1}$, both being maximal compact subgroups of $G^{e}$, are conjugate by an element $h \in G^{e}$, replacing $g$ by $g h$ if necessary we can as well assume that $g K^{e} g^{-1}=K^{e}$. By the same argument, in fact, we can also assume that

(i) $g K^{e} g^{-1}=K^{e}, \operatorname{Ad}_{\mathfrak{g}^{\mathbb{C}}}(g)(\mathfrak{k})=\mathfrak{k}, \operatorname{Ad}_{\mathfrak{g}^{\mathbb{C}}}(g)(\mathfrak{p})=\mathfrak{p}$ and

(ii) $\operatorname{Ad}_{\mathfrak{g}^{\mathbb{C}}}(g) \mathfrak{b}_{\mathfrak{k}}=\mathfrak{b}_{\mathfrak{k}}$,

where $\mathfrak{b}_{\mathfrak{k}}$ is a fixed Borel subalgebra of $\mathfrak{k}^{\mathbb{C}}$. The Borel subgroup of $K^{e, \mathbb{C}}$ corresponding to $\mathfrak{b}_{\mathfrak{k}}$ intersects $K^{e}$ in a compact Cartan subgroup $T^{e}$ of $K^{e}$. We note that in addition to (i) and (ii) above we also have

(iii) $T^{e}=g T^{e} g^{-1}$.

For later use we note that the above reductions imply:

Remark $\ddagger$ : If $N_{K}\left(T^{e}\right)$ is the normalizer of $T^{e}$ in $K$, then

$$
K=N_{K}\left(T^{e}\right) K^{e} \quad \text { and } \quad G=N_{K}\left(T^{e}\right) G^{e} .
$$

Let $\mathfrak{t}$ be the Lie algebra of $T^{e}$. Let $P_{\mathfrak{k}} \subseteq \Delta\left(\mathfrak{k}^{\mathbb{C}}, \mathfrak{t}^{\mathbb{C}}\right)$ be the positive system (in the set of compact roots) whose root spaces together with $\mathfrak{t}^{\mathbb{C}}$ span $\mathfrak{b}_{\mathfrak{k}}$.

Let $W_{\mathfrak{k}^{\mathbb{C}}}=W\left(K^{e}, T^{e}\right)=N_{K^{e}}\left(T^{e}\right) / T^{e}$ be the Weyl group of $K^{e}$. Here $N_{K^{e}}\left(T^{e}\right)$ is the normalizer of $T^{e}$ in $K^{e}$. Suppose that $\omega_{G^{e}}=\varpi(\Lambda)$ where $\varpi(\Lambda)$ is the discrete series representation of $G^{e}$ corresponding to $P_{\mathfrak{k}}$-dominant Harish-Chandra parameter $\Lambda$ which is $\Delta\left(\mathfrak{g}^{\mathbb{C}}, \mathfrak{t}^{\mathbb{C}}\right)$-regular. Let $P \subseteq \Delta\left(\mathfrak{g}^{\mathbb{C}}, \mathfrak{t}^{\mathbb{C}}\right)$ be the unique positive system of roots in $\Delta\left(\mathfrak{g}^{\mathbb{C}}, \mathfrak{t}^{\mathbb{C}}\right)$ defined by $P=\left\{\alpha \in \Delta\left(\mathfrak{g}^{\mathbb{C}}, \mathfrak{t}^{\mathbb{C}}\right) \mid(\Lambda, \alpha)>0\right\}$. Write $\rho=$ $\frac{1}{2} \sum_{\alpha \in P} \alpha$. The action of $\left.\varpi(\Lambda)\right|_{C}$ 。 is scalar multiplication by a unitary character with differential $\left.(\Lambda-\rho)\right|_{\mathfrak{c}}$. Thus $\left.(\Lambda-\rho)\right|_{\mathfrak{c}}$ takes values in $2 \pi \sqrt{-1} \mathbb{Z}$ on the lattice in $\mathfrak{c}$ which is the kernel of the exponential map from $\mathfrak{c}$ to the identity component of $C^{\circ}$. Furthermore, $\left.\varpi(\Lambda)\right|_{G_{1}}$ is the same as $\varpi\left(\left.\Lambda\right|_{\mathfrak{t} \cap \mathfrak{g}_{1}}\right)$ in the usual Harish-Chandra discrete series parametrization for $G_{1}$.

Let $\mathfrak{b}_{\mathfrak{g}}$ be the Borel sualgebra of $\mathfrak{g}^{\mathbb{C}}$ spanned by $\mathfrak{t}^{\mathbb{C}}$ and the root spaces corresponding to roots in $P$. We write $\mathfrak{b}^{\prime}=\operatorname{Ad}_{\mathfrak{g}^{\mathbb{C}}}(g)\left(\mathfrak{b}_{\mathfrak{g}}\right)$. In addition to (i), (ii) and (iii) above we also have

$$
\text { (iv) } \begin{aligned}
\mathfrak{b}^{\prime} & =\operatorname{Ad}_{\mathfrak{g}^{\mathbb{C}}}(g)\left(\mathfrak{b}_{\mathfrak{g}}\right)=\operatorname{Ad}_{\mathfrak{g}^{\mathbb{C}}}(g)\left(\mathfrak{b}_{\mathfrak{g}} \cap \mathfrak{k}^{\mathbb{C}}\right) \oplus \operatorname{Ad}_{\mathfrak{g}^{\mathbb{C}}}(g)\left(\mathfrak{b}_{\mathfrak{g}} \cap \mathfrak{p}^{\mathbb{C}}\right) \\
& =\mathfrak{b}_{\mathfrak{k}} \oplus \operatorname{Ad}_{\mathfrak{g}^{\mathbb{C}}}(g)\left(\mathfrak{b}_{\mathfrak{g}} \cap \mathfrak{p}^{\mathbb{C}}\right) .
\end{aligned}
$$

Let $P^{\prime}$ be the positive system of roots in $\Delta\left(\mathfrak{g}^{\mathbb{C}}, \mathfrak{t}^{\mathbb{C}}\right)$ corresponding to the Borel subalgebra $\mathfrak{b}^{\prime}$ of $\mathfrak{g}$. Note that $P^{\prime} \supset P_{\mathfrak{k}}$. In the $W\left(\mathfrak{g}^{\mathbb{C}}, \mathfrak{t}^{\mathbb{C}}\right)$-orbit of $\Lambda$ choose the unique linear form $\Lambda^{\prime}$ dominant with respect to $P^{\prime}$. Then $\varpi^{g}(\Lambda)=\varpi\left(\Lambda^{\prime}\right)$. This implies that $\varpi^{g}(\Lambda)$ and $\varpi(\Lambda)$ are isomorphic if and only if $\mathfrak{b}=\mathfrak{b}^{\prime}$. Since by (4.1) $\operatorname{Ad}_{\mathfrak{g}^{\mathbb{C}}}(g)$ is also the inner conjugation by $\Psi(g) \in I\left(\mathfrak{g}^{\mathbb{C}}\right)$ (the connected complex adjoint group of $\mathfrak{g}^{\mathbb{C}}$ ) on $\mathfrak{g}^{\mathbb{C}}$ the facts

$$
\begin{aligned}
& \operatorname{Ad}_{\mathfrak{g}^{\mathbb{C}}}(g)(\mathfrak{b})=\mathfrak{b}^{\prime}=\mathfrak{b} \text { and } \\
& g T^{e} g^{-1}=T^{e}
\end{aligned}
$$

imply $g \in \Psi^{-1}\left(\Psi\left(T^{e}\right)\right)=T^{e} \Psi^{-1}\left(\Psi\left(e_{G}\right)\right)=B T^{e} \subseteq B G^{e}$. This contradicts that we started with $g \in G \backslash B G^{e}$. This completes the proof of Proposition 4.7.

4.8. Fix a compact Cartan subgroup $T^{e} \subseteq K^{e}$, let $\mathfrak{t} \subseteq \mathfrak{k}, \mathfrak{t}^{\mathbb{C}} \subseteq \mathfrak{k}^{\mathbb{C}}$ denote the corresponding Lie subalgebras and let $\mathfrak{b}_{\mathfrak{k}} \subseteq \mathfrak{k}^{\mathbb{C}}$ be a Borel subalgebra containing $\mathfrak{t}^{\mathbb{C}}$. Let $\mathfrak{b} \subseteq \mathfrak{g}^{\mathbb{C}}$ be a Borel subalgebra of $\mathfrak{g}^{\mathbb{C}}$ containing $\mathfrak{b}_{\mathfrak{k}}$. Choose positive systems 
$P_{\mathfrak{k}} \subseteq \Delta\left(\mathfrak{k}^{\mathbb{C}}, \mathfrak{t}^{\mathbb{C}}\right)$ and $P \subseteq \Delta\left(\mathfrak{g}^{\mathbb{C}}, \mathfrak{t}^{\mathbb{C}}\right)$ containing $P_{\mathfrak{k}}$ corresponding to $\mathfrak{b}_{\mathfrak{k}}$ and $\mathfrak{b}$. Let $\varpi(\Lambda)$ be a discrete series representation of $G^{e}$ with Harish-Chandra parameter $\Lambda$ dominant with respect to $P$. Write $\Lambda=\lambda+\rho$ where $\rho=\frac{1}{2} \sum_{\alpha \in P} \alpha$. We also write $\rho_{\mathfrak{k}}=\frac{1}{2} \sum_{\alpha \in P_{\mathfrak{k}}} \alpha, \rho_{n}=\frac{1}{2} \sum_{\alpha \in P_{n}} \alpha$, where $P_{n}=P \backslash P_{\mathfrak{k}}$ is the set of positive noncompact roots. Let $\tau_{\lambda+2 \rho_{n}}$ be the finite-dimensional irreducible representation of $\mathfrak{k}^{\mathbb{C}}$ with $P_{\mathfrak{k}}$ highest weight $\lambda+2 \rho_{n}$. We use the same symbol $\tau_{\lambda+2 \rho_{n}}$ to denote the corresponding (group) representations of $K^{e}$ and $K^{e, \mathbb{C}}$. The representation $\left.\tau_{\lambda+2 \rho_{n}} \subseteq_{K^{e}} \varpi(\Lambda)\right|_{K^{e}}$. The group $C^{\circ}$ is the center of $G^{e}$ and $\left.C^{\circ} \subseteq K^{e} \cdot \varpi(\Lambda)\right|_{C^{\circ}}$ acts by the same unitary character as does $\left.\tau_{\lambda+2 \rho_{n}}\right|_{C^{\circ}}$. Let $\nu$ be an irreducible finite-dimensional representation of $B$ such that $\left.\nu\right|_{C^{\circ}}$ and $\tau_{\lambda+2 \rho_{n}} \mid C^{\circ}$ act by the same character. Then the tensor product $\nu \bigotimes \tau_{\lambda+2 \rho_{n}}$ is an irreducible representation of $B \times K^{e}$. The subgroup $\left\{\left(z, z^{-1}\right) \mid z \in C^{\circ}\right\}$ of $B \times K^{e}$ is in the kernel of $\nu \otimes \tau_{\lambda+2 \rho_{n}}$. Hence $\nu \bigotimes \tau_{\lambda+2 \rho_{n}}$ is in fact an irreducible representation of the quotient group $B K^{e}$.

Proposition 4.9. The induced representation $\mathcal{I}_{B K^{e}}^{K}\left(\nu \otimes \tau_{\lambda+2 \rho_{n}}\right)$ is irreducible.

Proof. The proof is similar to the proof of Proposition 4.7. By Mackey's criterion $\mathcal{I}_{B K^{e}}^{K}\left(\nu \otimes \tau_{\lambda+2 \rho_{n}}\right)$ is irreducibe if and only if $\forall g \in K \backslash B K^{e}$ the representations $\nu \otimes \tau_{\lambda+2 \rho_{n}}$ and $\left(\nu \otimes \tau_{\lambda+2 \rho_{n}}\right)^{g}$ are nonisomorphic. Replacing if necessary $g$ by $g h$ where $h \in K^{e}$ we can assume that $\mathfrak{b}_{\mathfrak{k}}^{g}=\mathfrak{b}_{\mathfrak{k}}$. Then $g T^{e} g^{-1}=T^{e}$. Define the Borel subalgebra $\mathfrak{b}^{\prime}=\mathfrak{b}^{g}$ of $\mathfrak{g}^{\mathbb{C}}$ as in the proof of Proposition 4.7. Let $P_{\mathfrak{k}}^{\prime}, P_{n}^{\prime}, \rho_{\mathfrak{k}}^{\prime}, \rho_{n}^{\prime}, \rho^{\prime}$ denote for $P^{\prime}$ what $P_{\mathfrak{k}}, P_{n}, \rho_{\mathfrak{k}}, \rho_{n}, \rho$ represent for $P$. Since $\mathfrak{b}_{\mathfrak{k}}^{g}=\mathfrak{b}_{\mathfrak{k}}$ we have $P_{\mathfrak{k}}^{\prime}=P_{\mathfrak{k}}$. We note that $\tau_{\lambda+2 \rho_{n}}^{g}=\tau_{\Lambda+\rho-2 \rho_{\mathfrak{k}}}^{g}=\tau_{\Lambda^{\prime}+\rho^{\prime}-2 \rho_{\mathfrak{k}}^{\prime}}$ where $\Lambda^{\prime}$ is the unique element in the $W\left(\mathfrak{g}^{\mathbb{C}}, \mathfrak{t}^{\mathbb{C}}\right)$ orbit of $\Lambda$ dominant with respect to $P^{\prime}$. In order to have $\tau_{\lambda+2 \rho_{n}}^{g}=\tau_{\lambda+2 \rho_{n}}$ we must have $\Lambda+\rho-2 \rho_{\mathfrak{k}}=\Lambda^{\prime}+\rho^{\prime}-2 \rho_{\mathfrak{k}}$. This is possible only if $P=P^{\prime}$. As in the proof of Proposition 4.7 this is possible only if $g \in B K^{e}$. This contradicts that we started with $g \in K \backslash B K^{e}$. This completes the proof of Proposition 4.9.

4.10. The main ingredient of the proof of Propositions 4.7 and 4.9 has some other important consequences which we explain. Let $\mathrm{J}_{B, \nu}$ be the finite-dimensional Hilbert space on which the representation $\nu$ of $B$ acts. With notation as in the proof of 4.7 and $4.9,\left.\left\{\mathcal{I}_{B G^{e}}^{G}(\nu \otimes \varpi(\Lambda))\right\}\right|_{B G^{e}}$ decomposes as $\oplus_{g}\left(\nu^{g} \nabla \varpi^{g}(\Lambda)\right)$, where the sum runs over coset representatives for $G / B G^{e}$. The $B G^{e}$-isomorphism class of $\nu^{g} \otimes \varpi^{g}(\Lambda)$ depends only on the coset $g B G^{e}$. Each $B G^{e}$-summand $\nu^{g} \nabla \varpi^{g}(\Lambda)$ is a discrete series representation of $B G^{e} . \mathcal{I}_{B G^{e}}^{G}(\nu \bigotimes \varpi(\Lambda))$ occurs in $\mathcal{I}_{B G^{e}}^{G}\left(\nu^{g} \bowtie \varpi^{g}(\Lambda)\right)$ (by Frobenius reciprocity). But the latter is also an irreducible $G$ module, by 4.7, hence both are $G$-isomorphic. For $h \in G \backslash B G^{e}, \nu^{g} \otimes \varpi^{g}(\Lambda)$ and $\left(\nu^{g} \otimes \varpi^{g}(\Lambda)\right)^{h}$ are nonisomorphic as $B G^{e}$-modules as seen in the proof of 4.7. In particular, $\left.\left\{\mathcal{I}_{B G^{e}}^{G}(\nu \otimes \varpi(\Lambda))\right\}\right|_{B G^{e}}$, as a $B G^{e}$ module is multiplicity free.

In the same way, $\left.\left\{\mathcal{I}_{B K^{e}}^{K}\left(\nu \otimes \tau_{\left(\lambda+2 \rho_{n}\right)}\right)\right\}\right|_{B K^{e}}$ decomposes as $\oplus_{g}\left(\nu^{g} \otimes \tau_{\left(\lambda+2 \rho_{n}\right)}^{g}\right)$, where the sum runs over coset representatives for $K / B K^{e}$; it is multiplicity free. In fact, $\mathcal{I}_{B K^{e}}^{K}\left(\nu \otimes \tau_{\left(\lambda+2 \rho_{n}\right)}\right)$ is isomorphic to $\mathcal{I}_{B K^{e}}^{K}\left(\nu^{g} \otimes \tau_{\left(\lambda+2 \rho_{n}\right)}^{g}\right)$. It may also be noted that $\varpi^{g}(\Lambda)=\varpi\left(\Lambda^{g}\right)$ and $\tau_{\left(\lambda+2 \rho_{n}\right)}^{g}=\tau_{\left(\lambda^{g}+2 \rho_{n}^{g}\right)}$ if we write $\Lambda^{g}, \rho^{g}$ for $\Lambda^{\prime}, \rho^{\prime}$ that we introduced in the proofs above.

Finally, if $\mathcal{I}_{B G^{e}}^{G}\left(\nu_{1} \otimes \varpi_{1}\right) \simeq_{G} \mathcal{I}_{B G^{e}}^{G}\left(\nu_{2} \otimes \varpi_{2}\right)$, then

$$
\left.\left.\mathcal{I}_{B G^{e}}^{G}\left(\nu_{1} \otimes \varpi_{1}\right)\right|_{B G^{e}} \simeq{ }_{B G^{e}} \mathcal{I}_{B G^{e}}^{G}\left(\nu_{2} \otimes \varpi_{2}\right)\right|_{B G^{e}}
$$


which from the above discussion implies $\nu_{2} \otimes \varpi_{2} \simeq_{B G^{e}} \nu_{1}^{g} \otimes \varpi_{1}^{g}$ for some $g \in G$. So, $\nu_{2} \simeq_{B} \nu_{1}^{g}$ and $\varpi_{2} \simeq_{G^{e}} \varpi_{1}^{g}$ for some $g \in G$.

4.11. Lemma. If for some nonempty subset $A \subseteq P_{n},\left(\lambda+2 \rho_{n}\right)-\langle A\rangle$ is $P_{\mathfrak{k}^{-}}$ dominant, then the $B K^{e}$-module $\left.\mathcal{I}_{B G^{e}}^{G}(\nu \otimes \varpi(\Lambda))\right|_{B K^{e}}$ does not have a $B K^{e}$ summand isomorphic to $\nu \bigotimes \tau_{\left(\lambda+2 \rho_{n}\right)-\langle A\rangle}$.

Proof. Applying Proposition 3.2.0 this follows from the discussion 4.10.

We continue with the notation introduced above. Thus $C^{\circ} \subseteq B$ and $B$ has trivial adjoint action on $\mathfrak{g}^{\mathbb{C}}$, in particular, on $\mathfrak{p}^{\mathbb{C}}$. Hence $C^{\circ}$ acts by the same unitary character both on $\mathfrak{p}^{\mathbb{C}} \otimes \mathrm{W}_{\left(\lambda+2 \rho_{n}\right)}$ and on $\mathrm{W}_{\left(\lambda+2 \rho_{n}\right)}$. Note that $\mathfrak{p}^{\mathbb{C}} \otimes \mathrm{W}_{\left(\lambda+2 \rho_{n}\right)}$ is a module for $K^{e}$ for the tensor product action $\left.\left(\operatorname{Ad}_{\mathfrak{g}^{\mathbb{C}}}\right)\right|_{\mathfrak{p}^{\mathbb{C}}} \otimes \tau_{\left(\lambda+2 \rho_{n}\right)}$. Let $\mathrm{H}_{\varpi, K^{e}}$ be the space of $K^{e}$ finite vectors in $\mathrm{H}_{\varpi}$, the Hilbert space on which the discrete series representaion $\varpi=\varpi(\Lambda)$ of $G^{e}$ acts. Then $\mathfrak{g}^{\mathbb{C}}$ acts on $\mathrm{H}_{\varpi, K^{e}}$, on $\mathrm{J}_{\nu} \otimes \mathrm{H}_{\varpi, K^{e}}$ (action of $\mathfrak{g}^{\mathbb{C}}$ on the second factor), and on $\mathcal{I}_{B G^{e}}^{G}(\nu \otimes \varpi)$. Choose an irreducible $K^{e}$ stable subspace of $\mathrm{H}_{\varpi, K^{e}}$ isomorphic to $\tau_{\left(\lambda+2 \rho_{n}\right)}$. We use the same notation $\mathrm{W}_{\left(\lambda+2 \rho_{n}\right)}$ for such a subspace.

4.12. Theorem. Let $\pi_{G}$ be an irreducible unitary representation of $G$ on a Hilbert space $\mathrm{H}_{\pi_{\mathrm{G}}}$. Let $\mathrm{H}_{\pi_{\mathrm{G}}, \mathrm{K}}$ be the space of $K$ finite vectors in $\mathrm{H}_{\pi_{\mathrm{G}}}$. Suppose that there exists an irreducible $K$-submodule $\mathcal{W} \subseteq \mathrm{H}_{\pi_{\mathrm{G}}, \mathrm{K}}$ which is $K$ isomorphic to an irreducible $K$ module $\mathcal{I}_{B K^{e}}^{K}\left(\nu \otimes \tau_{\left(\lambda+2 \rho_{n}\right)}\right)$ given by Proposition 4.9. We assume one of the following:

(a) If $\mathcal{T}$ is an irreducible $K$-submodule of $\mathfrak{p}^{\mathbb{C}} \otimes \mathcal{W}$ which does not occur in the discrete series $G$ representation $\left.\mathcal{I}_{B G^{e}}^{G}(\nu \otimes \varpi(\Lambda))\right|_{K}$ given by Proposition 4.7, then it does not occur in $\left.\pi_{G}\right|_{K}$, or

(b) The irreducible $B K^{e}$-module $\nu \otimes \tau_{\mu}$ does not occur in the $B K^{e}$-module $\pi_{G}\left(\mathfrak{p}^{\mathbb{C}}\right) \mathcal{W}$ if $\mu$ is $P_{\mathfrak{k}}$-dominant and $\mu=\left(\lambda+2 \rho_{n}\right)-\alpha$ where $\alpha \in P_{n}$.

Then $\pi_{G} \simeq_{G} \mathcal{I}_{B G^{e}}^{G}(\nu \otimes \varpi(\Lambda))$.

Proof. The hypothesis 4.12 (a) is conceptually slightly more elegant than 4.12 (b). Note, however, that if $\left(\lambda+2 \rho_{n}\right)+\alpha$, for some $\alpha \in P_{n}$, is the highest weight of an irreducible $\mathfrak{k}$-summand of $\mathfrak{p}^{\mathbb{C}} \otimes \mathrm{W}_{\left(\lambda+2 \rho_{n}\right)}$ which does not occur in $\varpi(\Lambda), 4.12$ (a) forbids an occurrence, while 4.12 (b) does not care.

For the proof of Theorem 4.12 under the hypothesis $4.12(\mathrm{~b})$ we need only a weaker condition to be satisfied: the $B K^{e}$-module $\pi_{G}\left(\mathfrak{p}^{\mathbb{C}}\right) \mathcal{W}$ does not have a $B K^{e}$ summand isomorphic to $\nu \otimes \tau_{\left(\lambda+2 \rho_{n}\right)-\alpha}$ for those $\alpha \in P_{n}$, such that $\left(\lambda+2 \rho_{n}\right)-\alpha$ is $P_{\mathfrak{k}}$-dominant and occurs as the highest weight of an irreducible $K^{e}$-summand in $\mathfrak{p}^{\mathbb{C}} \otimes \mathrm{W}_{\left(\lambda+2 \rho_{n}\right)}$.

The proof of Theorem 4.12 under the hypothesis 4.12 (a) runs almost parallel; towards the end we use Proposition 3.2.

The irreducible unitary representation $\pi_{G}$ of $G$ occurs as a $G$-summand in a representation $\mathcal{I}_{B G^{e}}^{G}\left(\pi_{B G^{e}}\right)$ of $G$ obtained by inducing from an irreducible $B G^{e}$ representation $\pi_{B G^{e}}$ on a Hilbert space $\mathrm{H}_{\pi_{B G} e}=\mathrm{J}_{\nu^{\prime}} \otimes \mathrm{H}_{\pi_{G} e}$, where $\mathrm{J}_{\nu^{\prime}}$ is the space on which $\left(B, \nu^{\prime}\right)$ acts and $\mathrm{H}_{\pi_{G e}}$ is the Hilbert space on which $\left(G^{e}, \pi_{G^{e}}\right)$ acts.

Let this inclusion be denoted by $\mathbf{i}: \mathrm{H}_{\pi_{G}, K} \rightarrow \mathcal{I}_{B G^{e}}^{G}\left(\pi_{B G^{e}}\right)$. If $v \in \mathrm{H}_{\pi_{G}, K}, \mathbf{i}(v)$ is a function on $G$ taking values in the space of smooth vectors in $\mathrm{H}_{\pi_{B G^{e}}}$. 
For $X \in \mathfrak{g}, v \in \mathrm{H}_{\pi_{G}, K}$, we note that $\mathbf{i}(X v)$ is given by differentiation of the function $\mathbf{i}(v)$ by the right invariant vector field $\mathbf{r}_{X}$ corresponding to $X$. Furthermore, $\mathbf{e v}_{\pi_{B G^{e}}}(\mathbf{i}(X v)) \in \mathrm{H}_{\pi_{B G^{e}}, B K^{e}}$, where $\mathrm{H}_{\pi_{B G^{e}}, B K^{e}}$ is the space of $B K^{e}$ finite vectors in $\mathrm{H}_{\pi_{B G^{e}}}$. We have $G=K B G^{e}$. The $K$-module $\left.\left\{\mathcal{I}_{B G^{e}}^{G}\left(\pi_{B G^{e}}\right)\right\}\right|_{K}$ is the same as $\mathcal{I}_{B K^{e}}^{K}\left(\left.\left(\pi_{B G^{e}}\right)\right|_{B K^{e}}\right)$ (using the Cartan decomposition $\left.G=K \exp (\mathfrak{p})\right)$. By the choice of $\pi_{B G^{e}}, \mathrm{H}_{\pi_{G}}, K \subseteq_{K} \mathcal{I}_{B K^{e}}^{K}\left(\left.\left(\pi_{B G^{e}}\right)\right|_{B K^{e}}\right)$. We have an evaluation map (from 4.5) $\mathbf{e v}_{\pi_{B G^{e}}}: \mathcal{I}_{B K^{e}}^{K}\left(\left.\left(\pi_{B G^{e}}\right)\right|_{B K^{e}}\right) \longrightarrow \mathrm{H}_{\pi_{B G^{e}}}$ which is $B K^{e}$-equivariant. The evaluation cannot be identically zero on a nonzero $K$-submodule of the induced module. Hence $\mathbf{e v}_{\pi_{B G e}}(\mathcal{W}) \neq 0$, for $\mathcal{W}$ as in the statement of the theorem. The $B K^{e}$ submodules of $\mathcal{W}$ are of the form $\nu^{g} \otimes \tau_{\lambda+2 \rho_{n}}^{g},(g \in K)$.

Under the adjoint action of $K, \mathfrak{g}^{\mathbb{C}}$ is a $K$-module, in particular, a $B K^{e}$ module. We have $\mathfrak{g}^{\mathbb{C}}=\mathfrak{k}^{\mathbb{C}} \oplus \mathfrak{p}^{\mathbb{C}}$ and $\mathfrak{p}^{\mathbb{C}}$ is a $K$-submodule. We have the following maps: By abuse of notation, we let the variable '?' in $\mathcal{I}_{B K^{e}}^{K}(?)$ stand both for a representation and for the space on which it acts:

$$
\begin{aligned}
& \mathcal{I}_{B K^{e}}^{K}\left(\mathrm{H}_{\pi_{B G^{e}}, B K^{e}}\right) \hookrightarrow \mathcal{I}_{B K^{e}}^{K}\left(\left.\left(\pi_{B G^{e}}\right)\right|_{B K^{e}}\right), \quad(K \text {-inclusion }), \\
& \mathbb{1}_{\mathfrak{p}^{\mathbb{C}}} \otimes \mathbf{i}: \mathfrak{p}^{\mathbb{C}} \otimes \mathcal{W} \rightarrow \mathfrak{p}^{\mathbb{C}} \otimes \mathcal{I}_{B K^{e}}^{K}\left(\left.\left(\pi_{B G^{e}}\right)\right|_{B K^{e}}\right), \quad(K \text {-equivariant }), \\
& \mathbf{r}: \mathfrak{p}^{\mathbb{C}} \otimes \mathcal{W} \rightarrow \mathcal{I}_{B K^{e}}^{K}\left(\left.\left(\pi_{B G^{e}}\right)\right|_{B K^{e}}\right), X \otimes v \mapsto \mathbf{r}_{X} v,(K \text {-equivariant }), \\
& \mathbf{r}^{e}: \mathfrak{p}^{\mathbb{C}} \otimes \mathrm{H}_{\pi_{B G^{e}}, B K^{e}}, \rightarrow \mathrm{H}_{\pi_{B G^{e}}, B K^{e}}, X \otimes v \mapsto \mathbf{r}_{X} v \text {, (BK } K^{e} \text {-equivariant), } \\
& \mathcal{I}\left(\mathbf{r}^{e}\right): \mathcal{I}_{B K^{e}}^{K}\left(\mathfrak{p}^{\mathbb{C}} \otimes \mathrm{H}_{\pi_{B G^{e}}, B K^{e}}\right) \rightarrow \mathcal{I}_{B K^{e}}^{K}\left(\mathrm{H}_{\pi_{B G^{e}}, B K^{e}}\right),(K \text {-equivariant }), \\
& \mathbf{e v}_{\mathrm{H}_{\pi_{B G}}, B K^{e}}: \mathcal{I}_{B K^{e}}^{K}\left(\mathrm{H}_{\pi_{B G^{e}}, B K^{e}}\right) \rightarrow \mathrm{H}_{\pi_{B G^{e}}, B K^{e}} \text {, (evaluation at } e, B K^{e} \text {-equivariant), } \\
& \mathfrak{p}^{\mathbb{C}} \otimes\left(\mathbf{e v}_{\pi_{B G^{e}}}\{\mathbf{i}(\mathcal{W})\}\right) \hookrightarrow \mathfrak{p}^{\mathbb{C}} \otimes \mathrm{H}_{\pi_{B G^{e}}, B K^{e}},\left(B K^{e} \text {-equivariant }\right),
\end{aligned}
$$

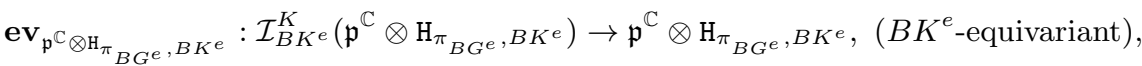

$$
\begin{aligned}
& \left.\mu_{\mathfrak{p} \mathbb{C}}: \mathfrak{p}^{\mathbb{C}} \otimes \mathcal{I}_{B K^{e}}^{K}\left(\mathrm{H}_{\pi_{B G^{e}}, B K^{e}}\right) \rightarrow \mathcal{I}_{B K^{e}}^{K}\left(\mathfrak{p}^{\mathbb{C}} \otimes \mathrm{H}_{\pi_{B G^{e}}, B K^{e}}\right) \text {, (K-equivalence, by } 4.5\right) \text {. }
\end{aligned}
$$

The following diagram is commutative:

$$
\begin{array}{ccc}
\mathfrak{p}^{\mathbb{C}} \otimes \mathrm{H}_{\pi_{B G^{e}}, B K^{e}} & \stackrel{\mathbf{r}^{e}}{\longrightarrow} & \mathrm{H}_{\pi_{B G^{e}}, B K^{e}} \\
\mathbf{e v}_{\mathfrak{p}^{\mathbb{C}} \otimes \mathrm{H}_{\pi_{B G}}, B K^{e}} \uparrow & \uparrow \mathbf{e v}_{\mathrm{H}_{\pi_{B G}}, B K^{e}} \\
\mathcal{I}_{B K^{e}}^{K}\left(\mathfrak{p}^{\mathbb{C}} \otimes \mathrm{H}_{\pi_{B G^{e}}, B K^{e}}\right) \stackrel{\mathcal{I}\left(\mathbf{r}^{e}\right)}{\longrightarrow} & \mathcal{I}_{B K^{e}}^{K}\left(\mathrm{H}_{\pi_{B G^{e}}, B K^{e}}\right)
\end{array}
$$

We also have the following commutative diagram in which both the vertical arrows are isomorphisms; the second one is in fact the identity map:

$$
\begin{array}{ccc}
\mathcal{I}_{B K^{e}}^{K}\left(\mathfrak{p}^{\mathbb{C}} \otimes \mathrm{H}_{\pi_{B G^{e}}, B K^{e}}\right) \stackrel{\mathcal{I}\left(\mathbf{r}^{e}\right)}{\longrightarrow} & \mathcal{I}_{B K^{e}}^{K}\left(\mathrm{H}_{\pi_{B G^{e}}, B K^{e}}\right) \\
\mu_{\mathfrak{p} \mathbb{C}} \uparrow & \uparrow \mathbb{1}_{\mathcal{I}} \\
\mathfrak{p}^{\mathbb{C}} \otimes \mathcal{I}_{B K^{e}}^{K}\left(\mathrm{H}_{\pi_{B G^{e}}, B K^{e}}\right) \stackrel{\mathbf{r}}{\longrightarrow} & \mathcal{I}_{B K^{e}}^{K}\left(\mathrm{H}_{\pi_{B G^{e}}, B K^{e}}\right)
\end{array}
$$

The map $\mathfrak{g}^{\mathbb{C}} \otimes \mathcal{W} \longrightarrow \mathcal{I}_{B K^{e}}^{K}\left(\left.\left(\pi_{B G^{e}}\right)\right|_{B K^{e}}\right)\left(=\left.\left\{\mathcal{I}_{B G^{e}}^{G}\left(\pi_{B G^{e}}\right)\right\}\right|_{K}\right), X \otimes v \mapsto \mathbf{r}_{X}(\mathbf{i}(v))$ is $K$-equivariant. 
4.13.1. It should be observed that since $\pi_{G}$ is a $G$-submodule of $\mathcal{I}_{B G^{e}}^{G}\left(\pi_{B G^{e}}\right)$, the image of the map $\mathfrak{g}^{\mathbb{C}} \otimes \mathcal{W} \longrightarrow \mathcal{I}_{B G^{e}}^{G}\left(\pi_{B G^{e}}\right)$ is a $K$-submodule of $\mathrm{H}_{\pi_{G}}, K$. The image of $\mathfrak{p}^{\mathbb{C}} \otimes \mathcal{W}$ is a $K$-submodule of the image of $\mathfrak{g}^{\mathbb{C}} \otimes \mathcal{W}$. It is the same as $\left(\mathcal{I}\left(\mathbf{r}^{e}\right) \circ \mu_{\mathfrak{p}^{\mathbb{C}}}\right)\left(\mathfrak{p}^{\mathbb{C}} \otimes \mathcal{W}\right) \subseteq K \mathrm{H}_{\pi_{G}}, K$

From our observation below the second commutative square in 4.5 we note that:

4.13.2.

(a) $\mathbf{r}^{e}\left\{\mathfrak{p}^{\mathbb{C}} \otimes\left(\mathbf{e v}_{\pi_{B G} e}\{\mathbf{i}(\mathcal{W})\}\right)\right\}=\left(\mathbf{e v}_{\pi_{B G}} \circ \mathcal{I}\left(\mathbf{r}^{e}\right)\right)\left\{\mu_{\mathfrak{p}^{\mathbb{C}}}\left(\mathfrak{p}^{\mathbb{C}} \otimes \mathcal{W}\right)\right\}$.

(b) From the assumption that $\mathcal{I}_{B K^{e}}^{K}\left(\nu \otimes \tau_{\lambda+2 \rho_{n}}\right)$ occurs in $\left.\left(\pi_{G}\right)\right|_{K}$ we conclude that for some $g \in K$, the irreducible $B K^{e^{e}}$-module $\nu^{g} \otimes \tau_{\lambda^{g}+2 \rho_{n}^{g}}$ occurs in $\mathbf{e v}_{\pi_{B G^{e}}}\{\mathbf{i}(\mathcal{W})\} \subseteq \mathrm{H}_{\pi_{B G^{e}}, B K^{e}}=\mathrm{J}_{\nu^{\prime}} \otimes \mathrm{H}_{\pi_{G^{e}}, K^{e}}$. In particular $\nu^{\prime}=\nu^{g}$ and $\tau_{\lambda+2 \rho_{n}}^{g}$ occurs in $\left.\pi_{G^{e}}\right|_{K^{e}}$. As in the discussions of $4.7,4.8$ and 4.9 , we will assume (by replacing $g$ in its coset $g B K^{e}$, if necessary) that $\operatorname{Ad}_{G^{e}} g$ fixes the compact Cartan subgroup $T^{e}$ of $K^{e}$ and the Borel subalgebra $\mathfrak{b}_{\mathfrak{k}} \subseteq \mathfrak{k}^{\mathbb{C}}$ containing $t$.

4.13.3. Recall from the beginning of the proof of 4.12 that $\pi_{B G^{e}}=\nu^{\prime} \otimes \pi_{G^{e}}=$ $\nu \otimes \pi_{G^{e}}$. Now suppose that $\pi_{G^{e}} \neq_{G^{e}} \varpi^{g}(\Lambda)$, where $g$ is as in 4.13 .2 (b).

Applying the companion theorem 1.1.1 to $\pi_{G^{e}}$ and $\varpi^{g}(\Lambda)$ we conclude that

1. For some noncompact root $\alpha \in P_{n},\left(\lambda^{g}+2 \rho_{n}^{g}\right)-\alpha^{g}$ is $P_{\mathfrak{k}}$-dominant. (This is the same as saying that $\left(\lambda+2 \rho_{n}\right)-\alpha$ is $P_{\mathfrak{k}}$-dominant since $\left.\operatorname{Ad}(g)\left(P_{\mathfrak{k}}\right)=P_{\mathfrak{k}}\right)$.

2. If $\left(\tau_{\left(\lambda^{g}+2 \rho_{n}^{g}\right)-\alpha^{g}}, \mathrm{~W}_{\left(\lambda^{g}+2 \rho_{n}^{g}\right)-\alpha^{g}}\right)$ is the $\mathfrak{k}^{-}$-representation with highest weight $\left(\lambda^{g}+2 \rho_{n}^{g}\right)-\alpha^{g}$, then the $B K^{e}$-module $\mathrm{J}_{\nu^{g}} \otimes \mathrm{W}_{\left(\lambda^{g}+2 \rho_{n}^{g}\right)-\alpha^{g}}$ occurs in

$$
\begin{aligned}
\pi_{B G^{e}}\left(\mathfrak{p}^{\mathbb{C}}\right)\left(\mathrm{J}_{\nu^{g}} \otimes \mathrm{W}_{\left.\lambda^{g}+2 \rho_{n}^{g}\right)}\right. & \subseteq B K^{e} \mathbf{r}^{e}\left\{\mathfrak{p}^{\mathbb{C}} \otimes\left(\mathbf{e v}_{\pi_{B G^{e}}}\{\mathbf{i}(\mathcal{W})\}\right)\right\} \\
& \subseteq_{B K^{e}} \mathrm{~J}_{\nu^{g}} \otimes \mathrm{H}_{\pi_{G^{e}}, K^{e}}
\end{aligned}
$$

Using 4.13.2 we now conclude that $\left(\mathbf{e v}_{\pi_{B G^{e}}} \circ \mathcal{I}\left(\mathbf{r}^{e}\right)\right)\left\{\mu_{\mathfrak{p}^{\mathbb{C}}}\left(\mathfrak{p}^{\mathbb{C}} \otimes \mathcal{W}\right)\right\}$ has a $B K^{e}$ type $\simeq \nu^{g} \otimes \tau_{\left(\lambda^{g}+2 \rho_{n}^{g}\right)-\alpha^{g}}$.

4.13.4. This requires that there exists a $K$-submodule $\mathcal{T}$ of $\left(\mathcal{I}\left(\mathbf{r}^{e}\right)\right)\left\{\mu_{\mathfrak{p}^{\mathbb{C}}}\left(\mathfrak{p}^{\mathbb{C}} \otimes \mathcal{W}\right)\right\}$ such that $\nu^{g} \otimes \tau_{\left(\lambda^{g}+2 \rho_{n}^{g}\right)-\alpha^{g}}$ occurs in $\left.\mathcal{T}\right|_{B K^{e}}$. Then

$$
\forall h \in K, \nu^{h} \otimes \tau_{\left(\lambda^{h}+2 \rho_{n}^{h}\right)-\alpha^{h}}
$$

is a $B K^{e}$-summand of $\left.\mathcal{T}\right|_{B K^{e}}$.

4.13.4 (b) If we are with hypothesis 4.12(b) this is straight away forbidden.

4.13.4 (a) Consider the above $K^{e}$-modules $\tau_{\left(\lambda^{h}+2 \rho_{n}^{h}\right)-\alpha^{h}}$. None of these $K^{e}$-modules occur in the $G^{e}$ discrete series representation $\varpi\left(\Lambda^{y}\right)$ for any $y \in G$ by Lemma 3.2. Consequently, from 4.10 none of the $B K^{e}$-modules $\nu^{h} \otimes \tau_{\left(\lambda^{h}+2 \rho_{n}^{h}\right)-\alpha^{h}}$ occur in $\left.\left\{\mathcal{I}_{B G^{e}}^{G}(\nu \otimes \varpi(\Lambda))\right\}\right|_{B K^{e}}$, the $G$-discrete series representation $\mathcal{I}_{B G^{e}}^{G}(\nu \otimes \varpi(\Lambda))$ regarded as a $B K^{e}$ module. Hence the $K$-module $\mathcal{T}$ does not occur in $\mathcal{I}_{B G^{e}}^{G}(\nu \otimes$ $\varpi(\Lambda))\left.\right|_{K}$. By hypothesis 4.12 (a) in Theorem 4.12, $\mathcal{T}$ does not occur in $\left.\left(\pi_{G}\right)\right|_{K}$. But this contradicts 4.13.4 and 4.13.1.

So, $\pi_{G^{e}} \simeq_{G^{e}} \varpi^{g}(\Lambda)$ and $\pi_{B G^{e}} \simeq_{B G^{e}} \nu^{g} \otimes \varpi^{g}(\Lambda)$ are both discrete series representations. The $G$-representation $\pi_{G}$ also belongs to the discrete series of $G$ since $\pi_{G}$ occurs as a $G$-summand in a representation $\mathcal{I}_{B G^{e}}^{G}\left(\nu^{g} \otimes \varpi^{g}(\Lambda)\right)$. The latter is irreducible (Proposition 4.7), and furthermore, from 4.10, $\mathcal{I}_{B G^{e}}^{G}\left(\nu^{h} \otimes \varpi^{h}(\Lambda)\right) \simeq_{G}$ $\mathcal{I}_{B G^{e}}^{G}(\nu \otimes \varpi(\Lambda)), \forall h \in G$. 
Conclusion: $\quad \pi_{G} \simeq_{G} \mathcal{I}_{B G^{e}}^{G}\{\nu \otimes \varpi((\Lambda))\}$.

This completes the proof of Theorem 4.12.

\section{5. $\mathbf{A}_{\mathfrak{q}}(\lambda)$}

For the rest of the article we assume $G=G^{e}$ is linear and of equal rank with finite center. In $\S \S 1,2$, and 3 we focussed on discrete series representations. We end by discussing briefly how some of the results of $\S \S 1,2$, and 3 can be generalized to the modules $A_{\mathfrak{q}}(\lambda)([18$, , 2], Theorem 5.3) Let $\mathfrak{q}=\mathfrak{l}+\mathfrak{u}$ be a parabolic subalgebra of $\mathfrak{g}^{\mathbb{C}}$ containing $\mathfrak{b}$. Let $\lambda$ be $P$-dominant integral and vanish on the roots of $\Delta\left(\mathfrak{l} \cap \mathfrak{p}^{\mathbb{C}}\right)$. Let $A=\sum_{\alpha \in \Delta(\mathfrak{u})} n_{\alpha} \alpha$ where $n_{\alpha}$ are nonnegative integers. Suppose that $\lambda+2 \rho\left(\mathfrak{u} \cap \mathfrak{p}^{\mathbb{C}}\right)+A$ is $P_{\mathfrak{k}^{-}}$dominant. Let $\left(\tau_{\lambda+2 \rho\left(\mathfrak{u} \cap \mathfrak{p}^{\mathbb{C}}\right)+A}, \mathrm{~W}_{\lambda+2 \rho\left(\mathfrak{u} \cap \mathfrak{p}^{\mathbb{C}}\right)+A}\right)$ be the $\mathfrak{k}$-module with highest weight $\lambda+2 \rho\left(\mathfrak{u} \cap \mathfrak{p}^{\mathbb{C}}\right)+A$.

5.0. Let $\xi$ be the $P_{\mathfrak{k}}$ highest weight of an irreducible $\mathfrak{k}$-summand of $\mathrm{W}_{\lambda+2 \rho(\mathfrak{u} \cap \mathfrak{p} \mathbb{C})+A} \otimes$ L. Then $\left(\xi+\rho_{\mathfrak{k}}, \xi+\rho_{\mathfrak{k}}\right) \geq\left(\lambda+\rho_{n}+\rho_{\mathfrak{k}}, \lambda+\rho_{n}+\rho_{\mathfrak{k}}\right)$. The inequality is strict if $A \neq 0$. This can be proved in the same way as [9], (4.8) whose proof ends with the last line of the paragraph containing ([9], eqn. 4.17). In particular when $A=\alpha$ the inequality is strict.

5.1. For a positive system $\check{P} \supset P_{\mathfrak{k}}$ we denote the PRV component of $\tau_{\lambda+2 \rho\left(\mathfrak{u} \cap \mathfrak{p}^{\mathbb{C}}\right)} \otimes \tau_{\check{\rho}_{n}}^{*}$ by $\tau_{\left[\lambda+2 \rho\left(\mathfrak{u} \cap \mathfrak{p}^{\mathbb{C}}\right)-\check{\rho}_{n}\right]}$. The notation $\left[\lambda+2 \rho\left(\mathfrak{u} \cap \mathfrak{p}^{\mathbb{C}}\right)-\check{\rho}_{n}\right]$ stands for the $P_{\mathfrak{k}}$-dominant weight in the $W\left(\mathfrak{k}^{\mathbb{C}}, \mathfrak{t}^{\mathbb{C}}\right)$-orbit of $\lambda+2 \rho\left(\mathfrak{u} \cap \mathfrak{p}^{\mathbb{C}}\right)-\check{\rho}_{n}$.

5.2. Consider the positive systems $\tilde{P}^{*}$ (notation from 9], see third line below 4.11, loc. cit $)$ in $\Delta\left(\mathfrak{g}^{\mathbb{C}}, \mathfrak{k}^{\mathbb{C}}\right)$ which contain both $P_{\mathfrak{k}}$ and $\Delta\left(\mathfrak{u} \cap \mathfrak{p}^{\mathbb{C}}\right)$. The inequality $\left(\xi+\rho_{\mathfrak{k}}, \xi+\rho_{\mathfrak{k}}\right) \geq\left(\lambda+\rho_{n}+\rho_{\mathfrak{k}}, \lambda+\rho_{n}+\rho_{\mathfrak{k}}\right)$ in (5.0) becomes an equality precisely when $A=0$ and $\tau_{\xi}$ is the PRV component $\tau_{\left[\lambda+2 \rho\left(\mathfrak{u} \cap \mathfrak{p}^{\mathbb{C}}\right)-\tilde{\rho}_{n}^{*}\right]}$ in $\tau_{\lambda+2 \rho\left(\mathfrak{u} \cap \mathfrak{p}^{\mathbb{C}}\right)} \otimes \tau_{\tilde{\rho}_{n}^{*}}^{*}$. We have

$$
\begin{gathered}
\left(\left[\lambda+2 \rho\left(\mathfrak{u} \cap \mathfrak{p}^{\mathbb{C}}\right)-\tilde{\rho}_{n}^{*}\right]+\rho_{\mathfrak{k}},\left[\lambda+2 \rho\left(\mathfrak{u} \cap \mathfrak{p}^{\mathbb{C}}\right)-\tilde{\rho}_{n}^{*}\right]+\rho_{\mathfrak{k}}\right) \\
=\left(\lambda+\rho_{n}+\rho_{\mathfrak{k}}, \lambda+\rho_{n}+\rho_{\mathfrak{k}}\right) .
\end{gathered}
$$

Based on these observations we derive the following analogues of Lemma 2.2, Corollary 2.3 and Theorem 1.1 with almost identical proofs.

Lemma 5.3. Let $\pi$ be an irreducible unitary representation of $G$. Let $\mathrm{W}_{\lambda+2 \rho\left(\mathfrak{u} \cap \mathfrak{p}^{\mathbb{C}}\right)}$ be a $K$-stable subspace of $\mathrm{H}_{\pi}$ such that the $K$-module $\mathrm{W}_{\lambda+2 \rho(\mathfrak{u} \cap \mathfrak{p})}$ is irreducible and is isomorphic to the finite-dimensional $K$-representation $\tau_{\lambda+2 \rho(\mathfrak{u} \cap \mathfrak{p})}$ with $P_{\mathfrak{k}}$-highest weight $\lambda+2 \rho\left(\mathfrak{u} \cap \mathfrak{p}^{\mathbb{C}}\right)$. Suppose that $\tau_{\mu}$ does not occur in $\left.\pi\right|_{K}$ if $\mu$ is $P_{\mathfrak{k}}$-dominant and $\mu=\lambda+2 \rho\left(\mathfrak{u} \cap \mathfrak{p}^{\mathbb{C}}\right)-\alpha$ where $\alpha \in \Delta\left(\mathfrak{q} \cap \mathfrak{p}^{\mathbb{C}}\right)$. Then:

i) For each positive system $\tilde{P}^{*}$ containing $P_{\mathfrak{k}} \cup \Delta(\mathfrak{u})$ there exists a $\mathfrak{k}$-stable subspace $\mathrm{W}_{\left[\lambda+2 \rho\left(\mathfrak{u} \cap \mathfrak{p}^{\mathbb{C}}\right)-\tilde{\rho}_{\mathfrak{n}}^{*}\right]}$ of $\mathrm{W}_{\lambda+2 \rho\left(\mathfrak{u} \cap \mathfrak{p}^{\mathbb{C}}\right)} \otimes L$ which is the PRV component of $\tau_{\lambda+2 \rho\left(\mathfrak{u} \cap \mathfrak{p}^{\mathbb{C}}\right)} \otimes \tau_{\tilde{\rho}_{n}^{*}}^{*}$,

$$
\left\|\left[\lambda+2 \rho\left(\mathfrak{u} \cap \mathfrak{p}^{\mathbb{C}}\right)-\tilde{\rho}_{n}^{*}\right]+\rho_{\mathfrak{k}}\right\|^{2}=\left(\lambda+\rho_{n}+\rho_{\mathfrak{k}}, \lambda+\rho_{n}+\rho_{\mathfrak{k}}\right) .
$$

ii) If $\mu$ is the $P_{\mathfrak{k}}$-highest weight of an irreducible $\mathfrak{k}$-submodule of $D_{\pi}\left(\mathrm{W}_{\lambda+2 \rho(\mathfrak{u} \cap \mathfrak{p} \mathbb{C})} \otimes \mathrm{L}\right)$, then

$$
\begin{gathered}
\left(\mu+\rho_{\mathfrak{k}}, \mu+\rho_{\mathfrak{k}}\right)>\left(\lambda+\rho_{n}+\rho_{\mathfrak{k}}, \lambda+\rho_{n}+\rho_{\mathfrak{k}}\right), \\
\mu \neq\left[\lambda+2 \rho\left(\mathfrak{u} \cap \mathfrak{p}^{\mathbb{C}}\right)-\tilde{\rho}_{n}^{*}\right] .
\end{gathered}
$$


5.4. Corollary. For each positive system $\tilde{P}^{*}$ containing $P_{\mathfrak{k}} \cup \Delta(\mathfrak{u})$ let $\mathrm{W}_{\left[\lambda+2 \rho\left(\mathfrak{u} \cap \mathfrak{p}^{\mathbb{C}}\right)-\tilde{\rho}_{\mathrm{n}}^{*}\right]}$

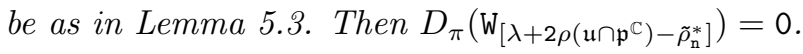

5.5. Theorem. Let $\pi$ be an irreducible unitary representation of $G$. Suppose that

(i) the finite-dimensional $K$-representation $\tau_{\lambda+2 \rho\left(\mathfrak{u} \cap \mathfrak{p}^{\mathbb{C}}\right)}$ with $P_{\mathfrak{k}}$-highest weight $\lambda+2 \rho\left(\mathfrak{u} \cap \mathfrak{p}^{\mathbb{C}}\right)$ occurs in $\left.\pi\right|_{K}$, and

(ii) $\tau_{\mu}$ does not occur in $\left.\pi\right|_{K}$ if $\mu$ is $P_{\mathfrak{k}}$-dominant and $\mu=\lambda+2 \rho\left(\mathfrak{u} \cap \mathfrak{p}^{\mathbb{C}}\right)-\alpha$ where $\alpha \in \Delta\left(\mathfrak{q} \cap \mathfrak{p}^{\mathbb{C}}\right)$.

Then $\pi$ is unitarily equivalent to $A_{\mathfrak{q}}(\lambda)$.

For proving Theorem 5.5 using 5.4 we proceed by starting exactly as in $\S \S 2$ and 3. First the infinitesimal character of $\pi$ is given by the orbit of $\lambda+\rho$. We arrive at an interim step as in 3.1 where initially we are able to conclude that:

5.5.1. There exists:

a parabolic subalgebra $\mathfrak{q}^{\prime}=\mathfrak{l}^{\prime}+\mathfrak{u}^{\prime}$ containing $\mathfrak{b}_{\mathfrak{k}}$,

a Borel subalgebra $\mathfrak{b}^{\prime} \subseteq \mathfrak{q}^{\prime}$ corresponging to a positive system $P^{\prime} \supset P_{\mathfrak{k}}$,

a $P^{\prime}$-dominant integral linear form $\lambda^{\prime}$ vanishing on $\Delta\left(\mathfrak{l}^{\prime} \cap \mathfrak{p}^{\mathbb{C}}\right)$ such that $\pi \simeq A_{\mathfrak{q}^{\prime}}\left(\lambda^{\prime}\right)$.

(See Remark 5.11 at the end.)

In particular, the infinitesimal character of $A_{\mathfrak{q}^{\prime}}\left(\lambda^{\prime}\right)$ is given by the orbit of $\lambda+\rho$. Hence, $\lambda+\rho$ and $\lambda^{\prime}+\rho^{\prime}$ both lie in the same $W\left(\mathfrak{g}^{\mathbb{C}}, \mathfrak{t}^{\mathbb{C}}\right)$-orbit. Since $\left.\tau_{\lambda+2 \rho(\mathfrak{u} \cap \mathfrak{p}} \mathbb{C}\right)$ occurs in $\left.\pi\right|_{\mathfrak{k}}=\left.A_{\mathfrak{q}^{\prime}}\left(\lambda^{\prime}\right)\right|_{\mathfrak{k}}$ from 3.1 (ii) we get

$$
\lambda+2 \rho\left(\mathfrak{u} \cap \mathfrak{p}^{\mathbb{C}}\right)=\lambda^{\prime}+2 \rho\left(\mathfrak{u}^{\prime} \cap \mathfrak{p}^{\mathbb{C}}\right)+\sum_{\beta \in \Delta\left(\mathfrak{u}^{\prime} \cap \mathfrak{p}^{\mathbb{C}}\right)} n_{\beta} \beta .
$$

On tensoring with $\mathrm{L}$ both $\tau_{\lambda+2 \rho\left(\mathfrak{u} \cap \mathfrak{p}^{\mathbb{C}}\right)}$ and $\tau_{\lambda^{\prime}+2 \rho\left(\mathfrak{u}^{\prime} \cap \mathfrak{p}^{\mathbb{C}}\right)}$ have a summand $\tau_{\xi}, \tau_{\xi}^{\prime}$, respectively, such that $\left(\xi+\rho_{\mathfrak{k}}, \xi+\rho_{\mathfrak{k}}\right)=\left(\lambda+\rho_{n}+\rho_{\mathfrak{k}}, \lambda+\rho_{n}+\rho_{\mathfrak{k}}\right)=\left(\lambda^{\prime}+\rho_{n}^{\prime}+\right.$ $\left.\rho_{\mathfrak{k}}, \lambda^{\prime}+\rho_{n}^{\prime}+\rho_{\mathfrak{k}}\right)=\left(\xi^{\prime}+\rho_{\mathfrak{k}}, \xi^{\prime}+\rho_{\mathfrak{k}}\right)$. This is possible only if $\sum_{\beta \in \Delta\left(\mathfrak{u}^{\prime} \cap p^{\mathbb{C}}\right)} n_{\beta} \beta$ is zero. So $\lambda+2 \rho\left(\mathfrak{u} \cap \mathfrak{p}^{\mathbb{C}}\right)=\lambda^{\prime}+2 \rho\left(\mathfrak{u}^{\prime} \cap \mathfrak{p}^{\mathbb{C}}\right)$. Write $\tau=\tau_{\lambda+2 \rho\left(\mathfrak{u} \cap \mathfrak{p}^{\mathbb{C}}\right)}=\tau_{\lambda^{\prime}+2 \rho\left(\mathfrak{u}^{\prime} \cap \mathfrak{p}^{\mathbb{C}}\right)}$

5.6. The positive systems $\check{P} \supset P_{\mathfrak{k}}$ of 5.1 for which we have equality in 5.2 are:

5.6.1. On the one hand, those which contain $P_{\mathfrak{k}} \cup \Delta\left(\mathfrak{u} \cap \mathfrak{p}^{\mathbb{C}}\right)$ if we think of $\tau$ as $\tau_{\lambda+2 \rho\left(\mathfrak{u} \cap \mathfrak{p}^{\mathbb{C}}\right)}$ (the $\check{P}$ here correspond to Borel subalgebras of $\mathfrak{q}$ containing $\mathfrak{b}_{\mathfrak{k}}$ ).

5.6.2. On the other hand, those which contain $P_{\mathfrak{k}} \cup \Delta\left(\mathfrak{u}^{\prime} \cap \mathfrak{p}^{\mathbb{C}}\right)$ if we think of $\tau$ as $\tau_{\lambda^{\prime}+2 \rho\left(\mathfrak{u}^{\prime} \cap \mathfrak{p}^{\mathbb{C}}\right)}$ (the $\check{P}$ here correspond to Borel subalgebras of $\mathfrak{q}^{\prime}$ containing $\mathfrak{b}_{\mathfrak{k}}$ ).

In particular, $\mathfrak{q} \cap \mathfrak{q}^{\prime}$ contains a Borel subalgebra of $\mathfrak{g}^{\mathbb{C}}$, hence $\mathfrak{q} \cap \mathfrak{q}^{\prime}$ is a parabolic subalgebra of $\mathfrak{g}^{\mathbb{C}}$. We write $\hat{\mathfrak{q}}$ for $\mathfrak{q} \cap \mathfrak{q}^{\prime}$.

5.7. The stabilizer $\tilde{\mathfrak{q}}_{\mathfrak{k}} \subseteq \mathfrak{k}$ of the line $\mathbb{C v} \subseteq \mathrm{W}_{\tau}$, where $\mathbf{v}$ is a nonzero $P_{\mathfrak{k}}$-highest weight vector in $W_{\tau}$ is a parabolic subalgebra $\tilde{\mathfrak{q}}_{\mathfrak{k}}=\tilde{\mathfrak{l}}_{\mathfrak{k}}+\tilde{\mathfrak{u}}_{\mathfrak{k}}$ of $\mathfrak{k}^{\mathbb{C}}$ containing $\mathfrak{b}_{\mathfrak{k}}$.

Define a positive system $P_{\mathfrak{k}}^{-} \subset \Delta\left(\mathfrak{k}^{\mathbb{C}}, \mathfrak{t}^{\mathbb{C}}\right)$ by $P_{\mathfrak{k}}^{-}=\Delta\left(\tilde{\mathfrak{u}}_{\mathfrak{k}}\right) \cup-\left\{P_{\mathfrak{k}} \cap \Delta\left(\tilde{\mathfrak{l}}_{\mathfrak{k}}\right)\right\}$. Define $P_{n}^{-} \subset \Delta\left(\mathfrak{p}^{\mathbb{C}}\right)$ by $P_{n}^{-}=\Delta\left(\mathfrak{u} \cap \mathfrak{p}^{\mathbb{C}}\right) \cup-\left\{P_{n} \cap \Delta(\mathfrak{l})\right\}$. Define $P_{n}^{\prime-} \subset \Delta\left(\mathfrak{p}^{\mathbb{C}}\right)$ by $P_{n}^{\prime-}=$ $\Delta\left(\mathfrak{u}^{\prime} \cap \mathfrak{p}^{\mathbb{C}}\right) \cup-\left\{P_{n}^{\prime} \cap \Delta\left(\mathfrak{l}^{\prime}\right)\right\}$.

Let $\rho_{n}^{-}=\frac{1}{2} \sum_{\alpha \in P_{n}^{-}} \alpha$ and let $\rho_{n}^{\prime-}=\frac{1}{2} \sum_{\alpha \in P_{n}^{\prime-}} \alpha$. Both are extreme weights of the spin module L.

5.8. Lemma. If $\alpha \in P_{\mathfrak{k}}$ and $\rho_{n}^{-}(\alpha)<0$, then $\left(\lambda+2 \Delta\left(\mathfrak{u} \cap \mathfrak{p}^{\mathbb{C}}\right)\right)(\alpha)=0$. If $\alpha \in P_{\mathfrak{k}}$ and $\rho_{n}^{\prime-}(\alpha)<0$, then $\left(\lambda^{\prime}+2 \Delta\left(\mathfrak{u}^{\prime} \cap \mathfrak{p}^{\mathbb{C}}\right)\right)(\alpha)=0$. 
5.9. Corollary. As a consequence $\rho_{n}^{-}$is $P_{\mathfrak{k}}^{-}$-dominant and $\rho_{n}^{\prime-}$ is $P_{\mathfrak{k}}^{-}$-dominant.

The idea of the proof of Lemma 5.8 is similar to that of $([9,3.34$; see for example [20], Lemma 2.2)

5.10. The observations 5.6, 5.6.1, and 5.6.2 can also be repeated substituting $P_{\mathfrak{k}}^{-}$ for $P_{\mathfrak{k}}$.

The set of noncompact roots contained in the intersection of all the positive systems $\check{P} \supset P_{\mathfrak{k}}$ appearing in 5.6 and the positive systems $\check{P}^{-} \supset P_{\mathfrak{k}}^{-}$appearing in 5.10 is, on the one hand, $\Delta\left(\mathfrak{u} \cap \mathfrak{p}^{\mathbb{C}}\right)$ if we think of $\tau$ as $\tau_{\lambda+2 \rho\left(\mathfrak{u} \cap \mathfrak{p}^{\mathbb{C}}\right)}$; whereas, on the other hand, it equals $\Delta\left(\mathfrak{u}^{\prime} \cap \mathfrak{p}^{\mathbb{C}}\right)$ if we think of $\tau$ as $\tau_{\lambda^{\prime}+2 \rho\left(\mathfrak{u}^{\prime} \cap \mathfrak{p}^{\mathbb{C}}\right)}$.

As a consequence, $\Delta\left(\mathfrak{u} \cap \mathfrak{p}^{\mathbb{C}}\right)=\Delta\left(\mathfrak{u}^{\prime} \cap \mathfrak{p}^{\mathbb{C}}\right),\left(\mathfrak{u} \cap \mathfrak{p}^{\mathbb{C}}\right)=\left(\mathfrak{u}^{\prime} \cap \mathfrak{p}^{\mathbb{C}}\right)$ and $\left(\mathfrak{q} \cap \mathfrak{p}^{\mathbb{C}}\right)=\left(\mathfrak{q}^{\prime} \cap\right.$ $\left.\mathfrak{p}^{\mathbb{C}}\right)$. Also, $2 \rho\left(\mathfrak{u} \cap \mathfrak{p}^{\mathbb{C}}\right)=2 \rho\left(\mathfrak{u}^{\prime} \cap \mathfrak{p}^{\mathbb{C}}\right)$. So ${ }^{\prime} \lambda+2 \rho\left(\mathfrak{u} \cap \mathfrak{p}^{\mathbb{C}}\right)=\lambda^{\prime}+2 \rho\left(\mathfrak{u}^{\prime} \cap \mathfrak{p}^{\mathbb{C}}\right) \Longrightarrow \lambda=\lambda$. Define $\overline{\mathfrak{q}}=\overline{\mathfrak{q}}_{\mathfrak{k}}+\overline{\mathfrak{q}}_{\mathfrak{p}}$, where $\overline{\mathfrak{q}}_{\mathfrak{p}}=\left(\mathfrak{q} \cap \mathfrak{p}^{\mathbb{C}}\right)$ and $\overline{\mathfrak{q}}_{\mathfrak{k}}=\left\{X \in \mathfrak{k}^{\mathbb{C}} \mid\left[X, \overline{\mathfrak{q}}_{\mathfrak{p}}\right] \subseteq \overline{\mathfrak{q}}_{\mathfrak{p}}\right\}$. Then $\overline{\mathfrak{q}}$ is a parabolic subalgebra of $\mathfrak{g}^{\mathbb{C}}$ containing $\mathfrak{q} \cup \mathfrak{q}^{\prime}$. The $\theta$-stable Levi part $\overline{\mathfrak{l}}$ of $\overline{\mathfrak{q}}$ may have some ideals contained in $\mathfrak{k}^{\mathbb{C}}$.

However, $A_{\mathfrak{q}}(\lambda)=A_{\overline{\mathfrak{q}}}(\lambda)=A_{\mathfrak{q}^{\prime}}(\lambda)=A_{\mathfrak{q}^{\prime}}\left(\lambda^{\prime}\right)=\pi$.

Remark 5.11. As a matter of fact, in the interim step 5.5.1 above, 2], Theorem 6.1 uses [11] to conclude something stronger: $\pi \simeq A_{\mathfrak{q}^{\prime}}\left(\lambda^{\prime}\right)$, where $\mathfrak{q}^{\prime} \supset \mathfrak{b}$ and $\lambda^{\prime}=\lambda$; if we had followed the same, some simplifications would have resulted. But we preferred the slightly longer argument here in the hope that perhaps it might simplify the proof of some earlier known results.

\section{REFERENCES}

[1] R. Hotta and R. Parthasarathy, Multiplicity formulae for discrete series, Invent. Math. 26 (1974), 133-178. MR0348041 (50 \#539)

[2] J.-S. Huang and P. Pandžić, Dirac cohomology, unitary representations and a proof of a conjecture of Vogan, J. Amer. Math. Soc. 15 (2002), no. 1, 185-202 (electronic), DOI 10.1090/S0894-0347-01-00383-6. MR1862801 (2002h:22022)

[3] J.-S. Huang and P. Pandžić, Dirac operators in representation theory, Mathematics: Theory \& Applications, Birkhäuser Boston, Inc., Boston, MA, 2006. MR2244116 (2007j:22025)

[4] B. Kostant, Lie algebra cohomology and the generalized Borel-Weil theorem, Ann. of Math. (2) 74 (1961), 329-387. MR0142696 (26 \#265)

[5] Harish-Chandra, On the theory of the Eisenstein integral, Conference on Harmonic Analysis (Univ. Maryland, College Park, Md., 1971), Springer, Berlin, 1972, pp. 123-149. Lecture Notes in Math., Vol. 266. MR0399355 (53 \#3200)

[6] Harish-Chandra, Harmonic analysis on real reductive groups. I. The theory of the constant term, J. Functional Analysis 19 (1975), 104-204. MR.0399356 (53 \#3201)

[7] K. R. Parthasarathy, R. Ranga Rao, and V. S. Varadarajan, Representations of complex semi-simple Lie groups and Lie algebras, Ann. of Math. (2) 85 (1967), 383-429. MR0225936 (37 \#1526)

[8] R. Parthasarathy, Dirac operator and the discrete series, Ann. of Math. (2) 96 (1972), 1-30. MR0318398 (47 \#6945)

[9] R. Parthasarathy, Criteria for the unitarizability of some highest weight modules, Proc. Indian Acad. Sci. Sect. A Math. Sci. 89 (1980), no. 1, 1-24. http://repository.ias.ac.in/82621/. MR.573381(82c:22020)

[10] R. Parthasarathy, A generalization of the Enright-Varadarajan modules, Compositio Math. 36 (1978), no. 1, 53-73. http://repository.ias.ac.in/82622/. MR515037 (80f:22016)

[11] S. A. Salamanca-Riba, On the unitary dual of real reductive Lie groups and the $A_{g}(\lambda)$ modules: the strongly regular case, Duke Math. J. 96 (1999), no. 3, 521-546, DOI 10.1215/S00127094-99-09616-3. MR,1671213(2000a:22023) 
[12] A. W. Knapp and D. A. Vogan Jr., Cohomological induction and unitary representations, Princeton Mathematical Series, vol. 45, Princeton University Press, Princeton, NJ, 1995. MR.1330919 (96c:22023)

[13] N. R. Wallach, Real reductive groups. I, Pure and Applied Mathematics, vol. 132, Academic Press, Inc., Boston, MA, 1988. MR929683 (89i:22029)

[14] V. S. Varadarajan, George Mackey and his work on representation theory and foundations of physics, Group representations, ergodic theory, and mathematical physics: a tribute to George W. Mackey, Contemp. Math., vol. 449, Amer. Math. Soc., Providence, RI, 2008, pp. 417-446, DOI 10.1090/conm/449/08722. MR2391814 (2009i:22005)

[15] V. S. Varadarajan, Some mathematical reminiscences, Methods Appl. Anal. 9 (2002), no. 3, v-xviii, DOI 10.4310/MAA.2002.v9.n3.a2. MR2023128(2005d:60001)

[16] A. Khare, Representations of complex semi-simple Lie groups and Lie algebras, Connected at infinity. II, Texts Read. Math., vol. 67, Hindustan Book Agency, New Delhi, 2013, pp. 85-129. arXiv:1208.0416v2 [math.RT]. MR3135123

[17] A. W. Knapp and E. M. Stein, Intertwining operators for semisimple groups. II, Invent. Math. 60 (1980), no. 1, 9-84, DOI 10.1007/BF01389898. MR582703 (82a:22018)

[18] D. A. Vogan Jr. and G. J. Zuckerman, Unitary representations with nonzero cohomology, Compositio Math. 53 (1984), no. 1, 51-90. MR.762307 (86k:22040)

[19] D. A. Vogan Jr., Unitarizability of certain series of representations, Ann. of Math. (2) 120 (1984), no. 1, 141-187, DOI 10.2307/2007074. MR750719 (86h:22028)

[20] S. Kumaresan, On the canonical $k$-types in the irreducible unitary $g$-modules with nonzero relative cohomology, Invent. Math. 59 (1980), no. 1, 1-11, DOI 10.1007/BF01390311. MR.575078 (83c:17011)

[21] W. Schmid, On a conjecture of Langlands, Ann. of Math. (2) 93 (1971), 1-42. MR.0286942 (44 \#4149)

[22] W. Schmid, Discrete series, Representation theory and automorphic forms (Edinburgh, 1996), Proc. Sympos. Pure Math., vol. 61, Amer. Math. Soc., Providence, RI, 1997, pp. 83-113, DOI 10.1090/pspum/061/1476494. MR1476494 (98k:22051)

[23] H. Hecht and W. Schmid, A proof of Blattner's conjecture, Invent. Math. 31 (1975), no. 2, 129-154. MR0396855 (53 \#715)

Raja Ramanna Fellow Bharathiar University Coimbatore

E-mail address: sarathy.math.tifr@gmail.com

$U R L$ : www.math.tifr.res.in/ sarathy 